\title{
Moduli Space of Supersymmetric Solitons and Black Holes in Five Dimensions
}

\author{
Veronika Breunhölder (D), James Lucietti $(\mathbb{D}$
}

School of Mathematics and Maxwell Institute of Mathematical Sciences, University of Edinburgh, The King's Buildings, Edinburgh, EH9 3FD, UK. E-mail: v.breunhoelder@ed.ac.uk; j.lucietti@ed.ac.uk

Received: 10 January 2018 / Accepted: 1 June 2018

Published online: 1 August 2018 - (C) The Author(s) 2018

\begin{abstract}
We determine all asymptotically flat, supersymmetric and biaxisymmetric soliton and black hole solutions to five-dimensional minimal supergravity. In particular, we show that the solution must be a multi-centred solution with a Gibbons-Hawking base. The proof involves combining local constraints from supersymmetry with global constraints for stationary and biaxisymmetric spacetimes. This reveals that the horizon topology must be one of $S^{3}, S^{1} \times S^{2}$ or a lens space $L(p, 1)$, thereby providing a refinement of the allowed horizon topologies. We construct the general smooth solution for each possible rod structure. We find a large moduli space of black hole spacetimes with noncontractible 2-cycles for each of the allowed horizon topologies. In the absence of a black hole, we obtain a classification of the known 'bubbling' soliton spacetimes.
\end{abstract}

\section{Contents}

1. Introduction . . . . . . . . . . . . . . . . . . . . . . . 472

2. Supersymmetric Solutions in Five Dimensions . . . . . . . . . . . . . . 475

2.1 Gibbons-Hawking base . . . . . . . . . . . . . . . . . 475

2.2 Supersymmetric and biaxisymmetric spacetimes . . . . . . . . . . . . 480

3. Near-Horizon Geometry . . . . . . . . . . . . . . . . . . . . . . . . . . . . 484

3.1 Orbit space metric . . . . . . . . . . . . . . . . . . . 485

3.2 Locally $S^{3}$ horizon . . . . . . . . . . . . . . . . . . . . 486

$3.3 S^{1} \times S^{2}$ horizon . . . . . . . . . . . . . . . . . 488

3.4 Horizon regularity and topology . . . . . . . . . . . . . . . . 490

3.5 Summary . . . . . . . . . . . . . . . . . . 493

4. Geometry and Topology of the Axes . . . . . . . . . . . . . . . . . . 494

4.1 Rod structure . . . . . . . . . . . . . . . . . . . . . . . . . . . . . . . . . . . . . 494

4.2 Geometry of the axes . . . . . . . . . . . . . . . . . . . . 496

4.3 Smoothness at corners of orbit space . . . . . . . . . . . . . . . . . 497

4.4 Summary .......................... 501 
5. Moduli Space of Soliton and Black Hole Solutions . . . . . . . . . . . . . 501

5.1 Classification theorem . . . . . . . . . . . . . . . . 501

5.2 Soliton solutions . . . . . . . . . . . . . . . . . 505

5.3 Single black hole solutions . . . . . . . . . . . . . . . . . 506

5.4 Multi black hole solutions . . . . . . . . . . . . . . . . . . 507

5.5 Physical properties . . . . . . . . . . . . . . . . . . . 508

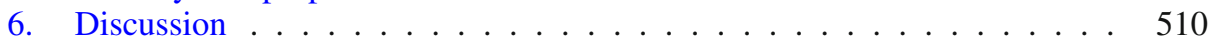

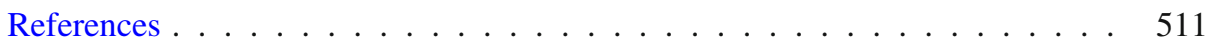

\section{Introduction}

The classification of isolated gravitating equilibrium states is a problem of central importance in general relativity. In four-dimensional Einstein-Maxwell theory this question has been answered, under some assumptions. Any asymptotically flat stationary spacetime must contain a black hole (no soliton theorem), and furthermore, the black hole uniqueness theorem implies it must be a Kerr-Newman solution, see e.g. [1].

For higher-dimensional general relativity, an analogous classification of equilibrium states is a major open problem [2]. It is known that both the no-soliton and the black hole uniqueness theorems are violated, even within the class of asymptotically flat (Minkowski $\mathbb{R}^{1, D-1}$ ) spacetimes. The failure of the uniqueness theorem was first revealed by the discovery of the black ring, an asymptotically flat stationary black hole vacuum solution with a horizon of spatial topology $S^{1} \times S^{2}$ [2]. It is now expected that the moduli space of stationary black hole solutions in higher dimensions is very rich, although further explicit solutions are hard to come by.

The failure of the no-soliton theorem became apparent after the construction of the 'bubbling' spacetimes in supergravity [3] (see [4] for a discussion of this). In particular, there exist finite energy, asymptotically flat, stationary spacetimes which are regular everywhere and contain no black hole region. The simplest examples are supersymmetric solutions to five-dimensional minimal supergravity (Einstein-Maxwell theory with a Chern-Simons coupling). Such spacetimes are topologically nontrivial and contain noncontracible 2-cycles, or 'bubbles', supported by magnetic flux. Indeed, such soliton spacetimes do not exist in vacuum gravity.

The existence of bubbling spacetimes leads to a further violation of black hole uniqueness. This is because one can envisage a black hole sitting in a bubbling spacetime. Indeed, the first law of black hole mechanics is modified by flux terms that couple to the bubbles [5]. Supersymmetric solutions describing a spherical black hole in an asymptotically flat bubbling spacetime can be constructed explicitly [6]. Interestingly, this leads to an entropy enigma which raises questions for the microscopic description of black holes in string theory [7]. Furthermore, these techniques have led to the construction of the first example of an asymptotically flat black hole with lens space topology, namely $L(2,1)$, termed a black lens [8]. Subsequently, black lenses with more general horizon topology $L(p, 1)$ have been constructed [9]. Thus, even in five-dimensional spacetimes, the moduli space of black hole solutions is now expected to be large and complicated.

A number of results have been derived that help constrain the topology and symmetry of asymptotically flat black hole spacetimes, for a review see [10]. Topological censorship implies the domain of outer communication (DOC) must be simply connected [11]. The horizon topology theorem states that spatial sections of the horizon admit positive scalar curvature, which in five dimensions only allows $S^{3}, S^{1} \times S^{2}, S^{3} / \Gamma$ and connected sums thereof [12]. The rigidity theorem implies that a stationary and rotating black hole 
must also be axisymmetric and thus possess an isometry group $\mathbb{R} \times U(1)$ [13]. Of course, these are all necessary conditions which must be satisfied; what is unclear is whether black hole solutions to Einstein's equations which realise all the above topology and symmetry constraints actually exist. The key question is: what is the moduli space of black hole solutions with a given topology and/or symmetry? There are essentially no results which address this question, except for static black hole solutions to Einstein-Maxwell theory for which a uniqueness theorem has been established [14-17].

In fact, the known explicit solutions possess more rotational symmetry than that guaranteed by the rigidity theorem. In particular, the five-dimensional solutions possess $\mathbb{R} \times U(1)^{2}$-symmetry and therefore belong to the class of generalised Weyl solutions $[18,19]$. As for four-dimensional stationary and axisymmetric spacetimes the Einstein equations become integrable and solutions can be classified in terms of a 'rod structure'. The rod structure is essentially a specification of how the $U(1)^{2}$-action degenerates on the axes of symmetry and given this data one can determine the spacetime and horizon topology. Indeed, by exploiting this structure a uniqueness theorem, which generalises the four-dimensional one, has been proven. This states that five-dimensional, asymptotically flat, stationary and biaxisymmetric vacuum black hole solutions are uniquely specified by their mass, angular momenta and rod structure [20,21]. This has been generalised to Einstein-Maxwell theory and minimal supergravity, where one finds that the magnetic flux on every noncontratible 2-cycle must also be specified [22-26]. ${ }^{1}$ However, in contrast to the four-dimensional case, the rod structure in five dimensions can be arbitrarily complicated in principle. What is not understood, is the existence problem: for what rod structures do suitably regular black hole solutions actually exist? Therefore, even the classification of five-dimensional stationary black holes with biaxial symmetry remains open (although see [31] for recent progress).

Of course, there are other types of symmetry assumptions which help simplify the construction of solutions. The classification of supersymmetric solutions has been well studied. Most of these works consist of local classifications. That is, deriving local constraints on the geometry arising from the existence of a suitable Killing spinor. However, what has been largely unstudied is a global analysis of supersymmetric solutions. Thus, a natural question presents itself: Can we classify all supersymmetric soliton and black hole solutions in five dimensions? Clearly, this requires a global analysis of suitably general supersymmetric solutions. Previously, a uniqueness theorem for supersymmetric spherical topology black holes in five-dimensional supergravity was proven [32,33], showing that the only solution was the BMPV black hole [34]. However, due to an overly restrictive assumption (the supersymmetric Killing field is strictly timelike in the DOC) it excluded the recently constructed black holes in bubbling spacetimes and black lenses $[6,8,9]$. The analogous result in four dimensions shows that the Majumdar-Papapetrou solutions are the most general supersymmetric black holes in Einstein-Maxwell theory [35].

In fact, the recent new examples of supersymmetric black hole solutions to fivedimensional supergravity all possess an $\mathbb{R} \times U(1)^{2}$-symmetry and hence are in the class of Weyl solutions (coupled to Maxwell field). Indeed, one can assign them a rod structure which thus demonstrates that solutions with more nontrivial rod structures do indeed exist in this case. Therefore, an even simpler question presents itself: can we classify all supersymmetric and biaxisymmetric soliton and black hole solutions in five dimensions? The purpose of this paper it is to show this is indeed possible.

\footnotetext{
1 Analogous results for asymptotically Kaluza-Klein solutions have also been obtained [27,28] (see also $[29,30])$.
} 
We will work in the simplest theory where such solutions exist, namely five-dimensional minimal supergravity. Supersymmetric solutions to this theory have been extensively studied [36]. Generically, they are timelike fibrations over a hyper-Kähler base. Remarkably, it was shown that if the base is a Gibbons-Hawking space, the full local form of the solution can be determined in terms of harmonic functions on an auxiliary $\mathbb{R}^{3}$. The known supersymmetric black hole solutions (including black rings $[37,38]$ and black lenses) and soliton solutions [39,40] belong to this class and are constructed from harmonic functions of multi-centred type.

One of our main results is the following classification theorem, the complete statement of which is given in Theorem 5 .

Theorem 1. Consider an asymptotically flat, supersymmetric and biaxisymmetric solution to five-dimensional minimal supergravity with a globally hyperbolic domain of outer communication, possibly containing a black hole. Then, the solution must have a Gibbons-Hawking base and the associated harmonic functions are of multi-centred type.

In the absence of a black hole, the above provides a classification of the bubbling soliton spacetimes in this symmetry class. This appears to be the first classification theorem known for such spacetimes. In the black hole case we find a rich moduli space of solutions, corresponding to bubbling spacetimes containing spherical black holes, black rings, or black lenses.

The proof consists of combining the local constraints from supersymmetry with global constraints for stationary and biaxisymmetric solutions. The main structure of the proof is as follows. As noted above supersymmetry determines the local form of the solution in terms of a set of harmonic functions on an auxiliary $\mathbb{R}^{3}$ [36]. This provides a key simplification which is not available in vacuum gravity. Thus, the proof reduces to a global analysis of this class of solutions. The structure of the orbit space of the domain of outer communication of Weyl solutions is that of a $2 \mathrm{~d}$ manifold, with a boundary which corresponds to horizons (if there is a black hole) or axes on which certain linear combinations of the biaxial Killing fields vanish, and corners where both biaxial Killing fields vanish [20,21]. Using the known classification of near-horizon geometries [32] allows us to prove that a smooth horizon corresponds to an isolated point on the boundary of the orbit space and furthermore shows that harmonic functions possess (at most) a simple pole at the horizon. Requiring smoothness of the DOC and the axes, together with some global constraints [41], also shows that the harmonic functions are nonsingular in the interior of the orbit space and everywhere else on its boundary, except at the corners where they possess simple poles. Thus, the number of simple poles the harmonic functions may possess is given by the number of horizons plus the number of corners of the orbit space.

We will also perform a detailed analysis of the possible rod structures and show that they are constrained by supersymmetry. In particular, this constrains the allowed horizon topologies to be one of $S^{3}, S^{1} \times S^{2}$ or a lens space $L(p, 1)$. Interestingly, this provides a refinement of the topologies allowed by biaxial symmetry; in particular it rules out $L(p, q)$ with $q \neq 1(\bmod p)$. Nevertheless, an infinite number of possible rod structures remains. We construct the explicit solution for every rod structure and determine the set of conditions required for the solution to be smooth and causal (on and outside a horizon). This reveals a very large moduli space of solutions both with and without a black hole and we give a general formula for the dimension of the moduli space. We find that for $n$-centred solutions, the number of inequivalent rod structures grows with $n$ and provide a formula for counting these for each horizon topology. 
The organisation of this paper is as follows. In Sect. 2 we examine local and global constraints on supersymmetric solutions imposed by the existence of biaxial symmetry commuting with supersymmetry. In Sects. 3 and 4 we examine the constraints imposed by the existence of a smooth event horizon and axes of symmetry, respectively. In Sect. 5 we present our main classification theorem and examine the moduli space of solutions. We end with a discussion in Sect. 6.

\section{Supersymmetric Solutions in Five Dimensions}

The bosonic sector of five-dimensional minimal supergravity consists of a spacetime metric $g$ and Maxwell field $F$ and the field equations are those for Einstein-Maxwell theory coupled to a Chern-Simons term. The general form of supersymmetric solutions of ungauged minimal supergravity is well understood [36]. Given a Killing spinor one can construct a smooth function $f$ and a Killing vector $V$, each quadratic in the spinor, such that $V \cdot V=-f^{2}$. Thus $V$ must be nonspacelike so the classification divides into solutions where $V$ is either null or timelike (at least in some region). The solutions where $V$ is null can be fully determined and correspond to plane wave and pp-wave spacetimes. We will be interested in asymptotically flat solutions, possibly containing a black hole, which must be in the timelike class.

In any region where the supersymmetric Killing field $V$ is timelike the spacetime metric takes the general form

$$
\mathrm{d} s^{2}=-f^{2}(\mathrm{~d} t+\omega)^{2}+f^{-1} h,
$$

where $V=\partial_{t}, h$ is a hyper-Kähler metric on the orthogonal space $B$ and $\omega$ is a 1-form on $B$. As we explain later, under the additional assumption of biaxial symmetry, the base must be a Gibbons-Hawking $(\mathrm{GH})$ space. It is thus convenient to first consider solutions with a GH base.

2.1. Gibbons-Hawking base. In this section we take the base metric to be a GibbonsHawking space, however we will not assume biaxial symmetry, so our analysis is valid in the general class of $\mathrm{GH}$ solutions. The $\mathrm{GH}$ metric is

$$
h=H^{-1}\left(\mathrm{~d} \psi+\chi_{i} \mathrm{~d} x^{i}\right)^{2}+H \mathrm{~d} x^{i} \mathrm{~d} x^{i},
$$

where $x^{i}, i=1,2,3$, are Cartesian coordinates on $\mathbb{R}^{3}$, the function $H$ is harmonic on $\mathbb{R}^{3}, \chi$ is a 1 -form on $\mathbb{R}^{3}$ satisfying

$$
\star 3 \mathrm{~d} \chi=\mathrm{d} H
$$

and $\partial_{\psi}$ is the triholomorphic Killing field.

As is well known, the local form of the supersymmetric solution can then be completely determined under the assumption that the full solution is invariant under the triholomorphic Killing field [36]. Such solutions are then specified by 4 harmonic functions $H, K, L, M$, in terms of which

$$
\begin{aligned}
f^{-1} & =H^{-1} K^{2}+L, \\
\omega & =\omega_{\psi}\left(\mathrm{d} \psi+\chi_{i} \mathrm{~d} x^{i}\right)+\hat{\omega}_{i} \mathrm{~d} x^{i},
\end{aligned}
$$


where

$$
\begin{aligned}
\omega_{\psi} & =H^{-2} K^{3}+\frac{3}{2} H^{-1} K L+M, \\
\star_{3} \mathrm{~d} \hat{\omega} & =H \mathrm{~d} M-M \mathrm{~d} H+\frac{3}{2}(K \mathrm{~d} L-L \mathrm{~d} K) .
\end{aligned}
$$

The Maxwell field is then

$$
F=d A=\frac{\sqrt{3}}{2} \mathrm{~d}\left[f(\mathrm{~d} t+\omega)-K H^{-1}\left(\mathrm{~d} \psi+\chi_{i} \mathrm{~d} x^{i}\right)-\xi_{i} \mathrm{~d} x^{i}\right],
$$

where the 1-form $\xi$ satisfies

$$
\star_{3} \mathrm{~d} \xi=-\mathrm{d} K \text {. }
$$

We wish to perform a global analysis of this family of local metrics. To this end, it will be useful to record the spacetime invariants

$$
\begin{aligned}
V \cdot V & =g_{t t}=-f^{2}=-\frac{H^{2}}{\left(K^{2}+H L\right)^{2}}, \\
\partial_{\psi} \cdot \partial_{\psi} & =g_{\psi \psi}=\frac{1}{f H}-f^{2} \omega_{\psi}^{2} \\
& =-\frac{4 H^{2} M^{2}+12 H K L M-4 H L^{3}+8 K^{3} M-3 K^{2} L^{2}}{4\left(H L+K^{2}\right)^{2}}, \\
V \cdot \partial_{\psi} & =g_{t \psi}=-f^{2} \omega_{\psi}=-\frac{H^{2} M+\frac{3}{2} H K L+K^{3}}{\left(K^{2}+H L\right)^{2}}, \\
A_{t} & =\frac{\sqrt{3}}{2} f, \quad A_{\psi}=\frac{\sqrt{3}}{2}\left(f \omega_{\psi}-\frac{K}{H}\right)=\frac{\sqrt{3}}{2} \frac{H M+\frac{1}{2} K L}{H L+K^{2}} .
\end{aligned}
$$

One can see that $A_{t}, A_{\psi}$ are invariants as follows. ${ }^{2}$ First note $\mathcal{L}_{V} F=\mathcal{L}_{\partial_{\psi}} F=0$ imply $\mathrm{d} \iota_{V} F=\mathrm{d} \iota_{\partial_{\psi}} F=0$. Therefore, for a simply connected spacetime (as we will be interested in) we deduce the existence of two globally defined functions $\Phi, \Psi$ satisfying

$$
\iota_{V} F=\frac{\sqrt{3}}{2} \mathrm{~d} \Phi, \quad \iota_{\partial_{\psi}} F=\frac{\sqrt{3}}{2} \mathrm{~d} \Psi .
$$

These functions $\Phi, \Psi$ are the electric potential and a magnetic potential respectively. From (8) we can identify these potentials up to an additive constant as $A_{t}=-\frac{\sqrt{3}}{2} \Phi$ and $A_{\psi}=-\frac{\sqrt{3}}{2} \Psi$, establishing these components of the gauge field are indeed spacetime invariants. Thus

$$
\Phi=-f, \quad \Psi=-f \omega_{\psi}+K H^{-1}
$$

(note the former is true even without a $\mathrm{GH}$ base). In terms of these invariants the solution is

$$
\begin{aligned}
\mathrm{d} s^{2}= & g_{t t}\left(\mathrm{~d} t+\hat{\omega}_{i} \mathrm{~d} x^{i}\right)^{2}+2 g_{t \psi}\left(\mathrm{d} t+\hat{\omega}_{i} \mathrm{~d} x^{i}\right)\left(\mathrm{d} \psi+\chi_{i} \mathrm{~d} x^{i}\right) \\
& +g_{\psi \psi}\left(\mathrm{d} \psi+\chi_{i} \mathrm{~d} x^{i}\right)^{2}+\frac{H}{f} \mathrm{~d} x^{i} \mathrm{~d} x^{i} \\
A= & A_{t}\left(\mathrm{~d} t+\hat{\omega}_{i} \mathrm{~d} x^{i}\right)+A_{\psi}\left(\mathrm{d} \psi+\chi_{i} \mathrm{~d} x^{i}\right)-\xi_{i} \mathrm{~d} x^{i} .
\end{aligned}
$$

${ }^{2}$ Smoothness of $A_{t}$ also follows from the fact that $f$ is a spacetime invariant (a bilinear in the Killing spinor). 
The inverse metric can be written as

$$
\begin{aligned}
g^{t t} & =-\frac{H}{f} g_{\psi \psi}+\frac{f}{H} \hat{\omega}_{i} \hat{\omega}_{i}, & g^{t i} & =-\frac{f}{H} \hat{\omega}_{i}, \\
g^{t \psi} & =\frac{H}{f} g_{t \psi}+\frac{f}{H} \hat{\omega}_{i} \chi_{i}, & g^{\psi i} & =-\frac{f}{H} \chi_{i}, \\
g^{\psi \psi} & =-\frac{H}{f} g_{t t}+\frac{f}{H} \chi_{i} \chi_{i}, & g^{i j} & =\frac{f}{H} \delta_{i j},
\end{aligned}
$$

and the determinant of the metric is

$$
\sqrt{-\operatorname{det} g}=\frac{H}{f}=K^{2}+H L .
$$

We now provide a spacetime interpretation of the harmonic functions.

First define the determinant of the matrix of inner products of the Killing fields $\partial_{t}, \partial_{\psi}$,

$$
N \equiv-\left|\begin{array}{cc}
g_{t t} & g_{t \psi} \\
g_{t \psi} & g_{\psi \psi}
\end{array}\right|
$$

This will be a key invariant in our analysis. From (10) it follows that

$$
N=\frac{f}{H} \Longrightarrow H=\frac{f}{N} \text {. }
$$

Next, we relate the harmonic functions $K, L, M$ to invariants as follows. From the above,

$$
K H^{-1}=\Psi-\frac{g_{t \psi}}{f} \Longrightarrow K=\frac{1}{N}\left(f \Psi-g_{t \psi}\right) .
$$

Using (4) and (6), together with the above expression for $K$ gives, after a little algebra,

$$
\begin{aligned}
L & =\frac{1}{N}\left(f g_{\psi \psi}+2 g_{t \psi} \Psi-f \Psi^{2}\right), \\
M & =\frac{1}{2 N}\left(g_{\psi \psi} g_{t \psi}-3 f \Psi g_{\psi \psi}-3 \Psi^{2} g_{t \psi}+f \Psi^{3}\right) .
\end{aligned}
$$

Observe that $K$ is only defined up to

$$
K \rightarrow K+c H
$$

where $c$ is a constant corresponding to the integration constant for $\Psi$. It follows that $L$, $M$ are defined up to the shifts

$$
L \rightarrow L-2 c K-c^{2} H, \quad M \rightarrow M-\frac{3}{2} c L+\frac{3}{2} c^{2} K+\frac{1}{2} c^{3} H .
$$

The following result will be useful for a global analysis of solutions with a GH base.

Lemma 1. Let $(g, F)$ be a supersymmetric solution to minimal supergravity with a Gibbons-Hawking base, $H, K, L, M$ the associated harmonic functions defined up to $(21,22)$, and let $N$ be defined as in (16).

1. If $H, K, L, M$ are smooth and

$$
K^{2}+H L>0
$$

then $(g, F)$ is smooth, $g^{-1}$ exists and is smooth, and $N>0$. 
2. Conversely, if $(g, F)$ is smooth and $N>0$, then $H, K, L, M$ are smooth and obey (23).

Proof. Smoothness of $H, K, L, M$ implies that the 1 -forms $\chi, \hat{\omega}, \xi$ must be smooth (otherwise their exterior derivatives $(3,7,9)$ would not be). Then, from (10), (13), (14) and (23) it follows that: (i) all components of the metric are smooth, (ii) a smooth inverse metric exists, and (iii) the Maxwell field is smooth. Furthemore, equation (17) shows that

$$
N^{-1}=K^{2}+H L,
$$

so (23) is equivalent to $N>0$. Therefore we have established part 1 of the Lemma. Part 2 immediately follows from $(17,18,19,20)$.

Remarks. 1. As we show in the next section, in the context of asymptotically flat, supersymmetric and biaxisymmetric spacetimes with a globally hyperbolic domain of outer communication, the invariant $N \geq 0$ on and outside any black hole region and vanishes only in two instances: (i) at fixed points of the triholomorphic Killing field $\partial_{\psi}=0$, or (ii) on an event horizon. We will analyse (i) and (ii) later making more detailed use of the biaxial symmetry together with certain global constraints.

2. We will require the spacetime to be stably causal,

$$
g^{t t}<0
$$

which means $t$ is a time function and is equivalent to the absence of CTC. In particular, as can be seen from (14), stable causality and $N>0$ imply that $g_{\psi \psi}>0$.

3. A priori, the metric in the chart $\left(t, \psi, x^{i}\right)$ is only defined in a region where $f \neq 0$, i.e. where $V$ is timelike. However, Lemma 1 shows that in the region $N>0$ the metric extends to a smooth solution even if $f=0$. In fact, (17) shows that in the region $N>0$, the zero set of $f$ is precisely the locus $H=0$. The zero set of $f$ is a smooth hypersurface in the spacetime if $\mathrm{d} f \neq 0$ everywhere on the zero set; ${ }^{3}$ then, from (17), it follows that in the region $N>0$ we may use the harmonic function $H$ as a coordinate in a neighbourhood of the zero set of $f$. In particular, we may introduce coordinates $\left(H, y^{A}\right)$ on $\mathbb{R}^{3}$ valid near $H=0$ so that

$$
\mathrm{d} x^{i} \mathrm{~d} x^{i}=\varrho^{2} \mathrm{~d} H^{2}+\tilde{g}_{A B} \mathrm{~d} y^{A} \mathrm{~d} y^{B} .
$$

The fact that $H$ is harmonic implies that $\sqrt{\operatorname{det} \tilde{g}}=\varrho F(y)$ where $F$ is an arbitrary function. By a coordinate change $y^{A} \rightarrow y^{\prime A}(y)$ we may arrange $\left.\sqrt{\operatorname{det} \tilde{g}}\right|_{H=0}=$ $F(y)$ so that $\varrho=\sqrt{\operatorname{det} \tilde{g}} /\left.\sqrt{\operatorname{det} \tilde{g}}\right|_{H=0}=1+O(H)$. The 1 -form $\chi$ now satisfies $\mathrm{d} \chi=\varrho^{-1} \tilde{\epsilon}$, where $\tilde{\epsilon}$ is the volume form of $\tilde{g}$, so $\chi=\left(\tilde{\chi}_{A}(y)+O(H)\right) \mathrm{d} y^{A}$ where $\mathrm{d} \tilde{\chi}=\left.\tilde{\epsilon}\right|_{H=0}$. The spacetime metric and gauge field induced on $H=0$ are

$$
\begin{aligned}
\left.\mathrm{d} s^{2}\right|_{H=0}= & -\frac{2}{K}\left(\mathrm{~d} t+\hat{\omega}_{A} \mathrm{~d} y^{A}\right)\left(\mathrm{d} \psi+\tilde{\chi}_{A} \mathrm{~d} y^{A}\right)+\frac{3 L^{2}-8 K M}{4 K^{2}}\left(\mathrm{~d} \psi+\tilde{\chi}_{A} \mathrm{~d} y^{A}\right)^{2} \\
& +K^{2} \tilde{g}_{A B} \mathrm{~d} y^{A} \mathrm{~d} y^{B}, \\
\left.A\right|_{H=0}= & \frac{\sqrt{3}}{2}\left(\frac{L}{2 K}\left(\mathrm{~d} \psi+\tilde{\chi}_{A} \mathrm{~d} y^{A}\right)-\xi_{A} \mathrm{~d} y^{A}\right) .
\end{aligned}
$$

\footnotetext{
${ }^{3}$ It is possible that the zero set of $f$ is not always a hypersurface; we will not analyse this possibility here. In any case, our analysis will not assume this.
} 
Note that in such a region (23) is satisfied, so $K$ must be non-vanishing at $H=0$. Thus we see that $H=0$ is a smooth timelike hypersurface. Therefore $H=0$ is an 'evanescent' ergosurface, i.e. a timelike hypersurface on which $f=0$ [42]. In fact, it has been shown [42] that any supersymmetric solution to minimal supergravity is smooth at an evanescent ergosurface if and only if the hyper-Kähler base is ambipolar (according to their definition) and $\omega$ has a particular behaviour near the ergosurface.

We will be interested in asymptotically flat solutions. For orientation, Minkowski space is given by

$$
H=\frac{1}{r}, \quad L=1, \quad K=M=0, \quad \chi=\cos \theta \mathrm{d} \phi, \quad \hat{\omega}=\xi=0,
$$

where we have written the $\mathbb{R}^{3}$ base in polar coordinates $(r, \theta, \phi)$. Upon the coordinate change $r=\rho^{2} / 4$ the metric is then

$$
\mathrm{d} s_{\text {Mink }}^{2}=-\mathrm{d} t^{2}+\mathrm{d} \rho^{2}+\frac{1}{4} \rho^{2}\left[(\mathrm{~d} \psi+\cos \theta \mathrm{d} \phi)^{2}+\mathrm{d} \theta^{2}+\sin ^{2} \theta \mathrm{d} \phi^{2}\right],
$$

so the spatial $\mathbb{R}^{4}$ is in polar coordinates with the round $S^{3}$ written in terms of Euler angles $(\theta, \phi, \psi)$. These are related to independently $2 \pi$-periodic angles $\phi^{ \pm}$in orthogonal planes via

$$
\phi^{ \pm}=\frac{1}{2}(\phi \mp \psi), \quad v_{ \pm} \equiv \partial_{\phi^{ \pm}}=\partial_{\phi} \mp \partial_{\psi} .
$$

Note $v_{+}=0$ on $\theta=0$ and $v_{-}=0$ on $\theta=\pi$ represent the two axes which extend out to infinity. In terms of Euler angles the periodicities are generated by the identifications $(\psi, \phi) \sim(\psi+4 \pi, \phi)$ and $(\psi, \phi) \sim(\psi+2 \pi, \phi+2 \pi)$.

The asymptotic expansion of an asymptotically flat spacetime is particularly simple for this class of metrics. Requiring that the harmonic functions $H, K, L, M$ asymptotically approach those of Minkowski spacetime (up to the freedom $(21,22)$ ) implies they can be written as a standard multipole expansion

$$
\begin{aligned}
H & =\frac{1}{r}+\sum_{l \geq 1, m} h_{l m} Y_{l m}(\theta, \phi) r^{-l-1} \\
K & =\frac{k_{\infty}}{r}+\sum_{l \geq 1, m} k_{l m} Y_{l m}(\theta, \phi) r^{-l-1}, \\
L & =1+\frac{\ell_{\infty}}{r}+\sum_{l \geq 1, m} \ell_{l m} Y_{l m}(\theta, \phi) r^{-l-1} \\
M & =m+\frac{m_{\infty}}{r}+\sum_{l \geq 1, m} m_{l m} Y_{l m}(\theta, \phi) r^{-l-1},
\end{aligned}
$$

where $k_{\infty}, \ell_{\infty}, m_{\infty}, m$ are constants. The constant $k_{\infty}$ is pure gauge and can be set to any value using (21). We have included a constant $m$ in $M$ in order not to fix the corresponding gauge freedom (22). The above form for the harmonic functions then determines the asymptotic expansion of the spacetime. In particular, this implies $f=1+O\left(r^{-1}\right)$ so the supersymmetric Killing vector $V$ is timelike near infinity, i.e. $V$ is the stationary Killing field. Furthermore, $\omega_{\psi}=m+\frac{3}{2} k_{\infty}+O\left(r^{-1}\right)$ so we set

$$
m=-\frac{3}{2} k_{\infty},
$$


which is indeed invariant under the gauge transformations (21) and (22). When integrating for $\chi$ and $\hat{\omega}$ we will also set the integration constants so that $\chi=\cos \theta \mathrm{d} \phi+O\left(r^{-1}\right)$ and $\hat{\omega}=O\left(r^{-1}\right)$. Without loss of generality, we may always make such choices for asymptotically flat solutions. It is now clear that the smoothness condition (23) and causality condition (25) are satisfied in the asymptotic region.

2.2. Supersymmetric and biaxisymmetric spacetimes. We now impose that a (timelike) supersymmetric background $(M, g, F)$ also has biaxial symmetry. As we will explain, this implies the solution must be of the type studied in the previous section (i.e. GibbonsHawking type).

In particular, we assume: (i) there is a $U(1)^{2}$-isometry, generated by Killing fields $m_{i}$, $i=1,2$, whose orbits are $2 \pi$ periodic; (ii) $\left[V, m_{i}\right]=0$ where $V$ is the supersymmetric Killing field; (iii) $\mathcal{L}_{m_{i}} F=0$. Clearly, the biaxial Killing fields are defined up to $m_{i} \rightarrow$ $A_{i j} m_{j}$ where $A_{i j}$ is an $S L(2, \mathbb{Z})$ matrix. We will sometimes denote the Killing fields collectively by $K_{A}$, where $A=0,1,2$ and $K_{0}=V$ and $K_{i}=m_{i}$.

The above conditions mean the spacetime is stationary and biaxisymmetric, where the supersymmetric Killing field $V$ is the stationary Killing field. In the context of (electro-)vacuum gravity these correspond to the well studied (generalised) Weyl solutions. Therefore we are dealing with supersymmetric Weyl solutions. The additional assumption of supersymmetry places extra local and global constraints on the solution which we will now explore.

Firstly, supersymmetry together with biaxial symmetry places strong constraints on the local form of the solution, as follows.

Lemma 2. Consider an asymptotically flat, supersymmetric and biaxisymmetric solution to minimal supergravity with supersymmetric Killing field $V=\partial_{t}$. Then the hyperKähler base must be a Gibbons-Hawking metric (2) whose triholomorphic Killing field $\partial_{\psi}$ leaves the full solution invariant. Furthermore, the harmonic functions $H, K, L, M$ on $\mathbb{R}^{3}$ are axisymmetric and the 1-forms can be written as ${ }^{4}$

$$
\chi=\chi(\rho, z) \mathrm{d} \phi, \quad \hat{\omega}=\hat{\omega}(\rho, z) \mathrm{d} \phi, \quad \xi=\xi(\rho, z) \mathrm{d} \phi,
$$

where $(\rho, z, \phi)$ are cylindrical coordinates on $\mathbb{R}^{3}$. In particular, in the coordinates $y^{A}=(t, \psi, \phi)$ and $z^{a}=(\rho, z)$, the spacetime metric (1) then takes the block diagonal form

$$
\mathrm{d} s^{2}=G_{A B}\left(z^{a}\right) \mathrm{d} y^{A} \mathrm{~d} y^{B}+q_{a b}\left(z^{a}\right) \mathrm{d} z^{a} \mathrm{~d} z^{b},
$$

where

$G_{A B} \mathrm{~d} y^{A} \mathrm{~d} y^{B}=-f^{2}\left(\mathrm{~d} t+\omega_{\psi}(\mathrm{d} \psi+\chi \mathrm{d} \phi)+\hat{\omega} \mathrm{d} \phi\right)^{2}+f^{-1} H^{-1}(\mathrm{~d} \psi+\chi \mathrm{d} \phi)^{2}+\frac{H \rho^{2}}{f} \mathrm{~d} \phi^{2}$

is the inner product on the space spanned by the Killing fields $\left\{\partial_{t}, \partial_{\psi}, \partial_{\phi}\right\}$, and

$$
q_{a b} \mathrm{~d} z^{a} \mathrm{~d} z^{b}=\frac{H}{f}\left(\mathrm{~d} \rho^{2}+\mathrm{d} z^{2}\right)
$$

\footnotetext{
4 For notational simplicity we denote both the 1 -forms and their $\phi$-components by $\chi, \hat{\omega}, \xi$. Distinction between these will be apparent from context, or clarified if necessary.
} 
is a metric on surfaces orthogonal to the space of Killing fields. The determinant of $G_{A B}$ is

$$
\operatorname{det} G_{A B}=-\rho^{2},
$$

so $(\rho, z)$ are in fact standard Weyl coordinates.

Proof. First we show that for a supersymmetric solution (1) biaxial symmetry requires the hyper-Kähler base $h$ and the data $f, \omega$ defined on it, to be $U(1)^{2}$-invariant. By assumption $\mathcal{L}_{m_{i}} V=0$ and therefore $f=\sqrt{-V_{\mu} V^{\mu}}$ implies $\mathcal{L}_{m_{i}} f=0$. Writing the hyper-Kähler metric as

$$
h_{\mu \nu}=f g_{\mu \nu}+f^{-1} V_{\mu} V_{\nu}
$$

we then immediately deduce $\mathcal{L}_{m_{i}} h_{\mu \nu}=0$. Finally, by a shift $t \rightarrow t+\lambda$, where $\lambda$ is a function on $B$, we may choose the time function so $\mathcal{L}_{m_{i}} t=0$, which also implies $\mathcal{L}_{m_{i}} \omega=0$ (by taking the Lie derivative of $V_{\mu} \mathrm{d} x^{\mu}=-f^{2}(\mathrm{~d} t+\omega)$ ).

We may now apply the following result [43]: A hyper-Kähler metric with a local $U(1)^{2}$-isometry is a Gibbons-Hawking metric and the triholomorphic Killing field is a linear combination of the $U(1)^{2}$ Killing fields. Therefore, biaxial symmetry of the full solution implies that the base must be a $\mathrm{GH}$ metric (2) and also that the full solution is invariant under the triholomorphic Killing field. Hence, the local form of the solution is determined by four harmonic functions $H, K, L, M$ on $\mathbb{R}^{3}$ as discussed in Sect.2.1.

The GH metrics in general only possess a $U(1)$-isometry generated by the triholomorphic Killing field. This allows us to write the harmonic function $H$ and the $\mathbb{R}^{3}$ base as the invariants $H^{-1}=h\left(\partial_{\psi}, \partial_{\psi}\right)$ and $\delta=H^{-1} h-h\left(\partial_{\psi}, \cdot\right)^{2}$ respectively. Hence, the $U(1)^{2}$-symmetry implies that $H$ and $\delta$ are invariant under a linear combination of the $U(1)^{2}$ Killing fields, say $\eta$, which is linearly independent to $\partial_{\psi}$. By shifting $\psi \rightarrow \psi+\lambda$, where $\lambda$ is a function on $\mathbb{R}^{3}$, we may set $\mathcal{L}_{\eta} \psi=0$. Then $\eta$ is a vector field on $\mathbb{R}^{3}$ which leaves $H$ and $\delta$ invariant, i.e. $\eta$ is a Killing vector of $\mathbb{R}^{3}$.

Hence we have shown that the $U(1)^{2}$-symmetry assumption implies the harmonic function $H$ is invariant under a 1-parameter group of isometries of $\mathbb{R}^{3}$. If this 1-parameter subgroup is closed then it must be a subgroup $U(1) \in S O(3)$, i.e. the harmonic function must be axisymmetric. What if $H$ is invariant under a non-compact 1-parameter subgroup of the Euclidean group? Such subgroups correspond to a translation, or a combination of a translation with a rotation (corkscrew). In either case, the orbit curves of such subgroups are unbounded in $\mathbb{R}^{3}$. Since $H$ is invariant along such an orbit curve, and by asymptotic flatness $H \rightarrow 0$ at infinity, we deduce that $H \equiv 0$ everywhere, a contradiction. This argument shows that the only 1-parameter subgroup of the Euclidean group which may leave $H$ invariant is the axial symmetry. Thus we write the $\mathbb{R}^{3}$ in cylindrical coordinates

$$
\mathrm{d} x^{i} \mathrm{~d} x^{i}=\mathrm{d} \rho^{2}+\rho^{2} d \phi^{2}+\mathrm{d} z^{2},
$$

where the axial Killing field is $\eta=\partial_{\phi}$. Then $H$ is invariant under $\partial_{\phi}$ and hence is only a function of $(\rho, z)$. We will now show that the other harmonic functions $K, L, M$ must also be axisymmetric, i.e. invariant under the axial Killing field $\partial_{\phi}$.

First, we recall some well known properties of Maxwell fields invariant under three commuting Killing fields $K_{A}$, see e.g. [5]. The Bianchi identity implies that the functions $\iota_{K_{A}} \iota_{K_{B}} F$ are constant in the spacetime. Furthermore, asymptotic flatness implies that a different linear combination of $K_{i}=m_{i}$, for $i=1,2$, vanish on the two axes which intersect the $S^{3}$ at infinity, namely $v_{+}$and $v_{-}$in (30). Therefore all these constants must in fact vanish, so $\iota_{K_{A}} \iota_{K_{B}} F=0$. In particular, since the axial Killing field $\partial_{\phi}$ and the triholomorphic Killing field $\partial_{\psi}$ must be linear combinations of the $K_{i}=m_{i}$ for $i=1,2$, 
we must have $\iota_{\partial_{\phi}} \iota_{\partial_{\psi}} F=0$ so by (11) the magnetic potential is axisymmetric $\mathcal{L}_{\partial_{\phi}} \Psi=0$. Hence (186) implies that $K H^{-1}$, and thus $K$, is also axisymmetric. Axisymmetry of $L$ and $M$ then follows from invariance of $f$ and $\omega_{\psi}$ under $\partial_{\phi}$, together with (4) and (6).

Furthermore, axisymmetry implies that the 1 -forms $\chi, \hat{\omega}, \xi$, are all gauge equivalent to (33). To see this, first note that axisymmetry of the harmonic function $H$ and invariance of the 1-form $h\left(\partial_{\psi}, \cdot\right)=H^{-1}(\mathrm{~d} \psi+\chi)$ under $\partial_{\phi}$, together with our gauge choice $\mathcal{L}_{\partial_{\phi}} \psi=0$, implies that $\mathcal{L}_{\partial_{\phi}} \chi=0$. Also, $0=\iota_{\partial_{\phi}} \mathrm{d} H=\iota_{\partial_{\phi}} \star_{3} \mathrm{~d} \chi \sim \rho^{2} \star_{3}(\mathrm{~d} \phi \wedge \mathrm{d} \chi)$, which implies $\chi=\chi(\rho, z) \mathrm{d} \phi+\mathrm{d}\left(\lambda^{\prime}(\rho, z)\right)$ for some function $\lambda^{\prime}(\rho, z)$. By a shift in $\psi \rightarrow \psi-\lambda^{\prime}$ (which does not affect $\mathcal{L}_{\partial_{\phi}} \chi=0$ ) we may eliminate $\lambda^{\prime}$. A similar argument works for the 1 -forms $\hat{\omega}$ and $\xi$ by shifting $t$ and the gauge field $A$ respectively, establishing the claim.

Putting everything together we find the spacetime metric can be written as claimed. The local form of the metric (34) shows the distribution orthogonal to $\operatorname{span}\left\{\partial_{t}, \partial_{\psi}, \partial_{\phi}\right\}$ is integrable so that at every point there exist surfaces orthogonal to the Killing fields with metric (36). ${ }^{5}$

We now turn to our global assumptions. We assume the spacetime $(M, g)$ is asymptotically flat and the domain of outer communication $\langle\langle M\rangle\rangle$ is globally hyperbolic so $\langle\langle M\rangle\rangle \cong \mathbb{R} \times \Sigma$. Topological censorship implies that $\langle\langle M\rangle\rangle$ is simply connected [11]. We will denote the event horizon by $\mathcal{H}$, although we allow for the possibility of no black hole region. We will assume the stationary Killing vector $V$ is complete so the spacetime has an isometry group $G=\mathbb{R} \times U(1)^{2}$, where $\mathbb{R}$ is tangent to the orbits of $V$. The axes correspond to the set of fixed points of the biaxial symmetry

$$
\mathcal{A}=\left\{p \in M \mid \operatorname{det} \gamma_{i j}(p)=0\right\}
$$

where $\gamma_{i j}=m_{i} \cdot m_{j}$ and $i, j=1,2$. Under these conditions, it has been shown that the orbit space

$$
\hat{M}=\langle\langle M\rangle\rangle / G \cong \Sigma / U(1)^{2}
$$

is a simply connected 2-dimensional manifold with boundaries and corners [10,20,21]. The axes corresponds to boundary segments $I \subset \partial \hat{M}$ where $\gamma_{i j}$ is rank- 1 and to corners of $\hat{M}$ where $\gamma_{i j}$ is rank-0. Below we will show that an event horizon, which must be degenerate, corresponds to a point on $\partial \hat{M}$ (in fact an asymptotic end, as is generic for extremal horizons, see e.g. [45]).

Now, we may identify the surfaces orthogonal to the Killing fields with the the orbit space $\hat{M}$. We deduce that the orbit space inherits the metric $q(36)$, so we will refer to this as the orbit space metric. Under the above global assumptions, it has been shown that $\operatorname{det} G_{A B}<0$ everywhere on $\langle\langle M\rangle\rangle \backslash \mathcal{A}$ and $\operatorname{det} G_{A B}=0$ on $\mathcal{H} \cup \mathcal{A}$ [41]. From (37) we immediately deduce that $\rho>0$ everywhere on $\langle\langle M\rangle\rangle \backslash \mathcal{A}$ and $\rho=0$ on $\mathcal{H} \cup \mathcal{A}$. Thus the interior of the orbit space corresponds to $\rho>0$ and its boundary to $\rho=0$. Therefore, $(\rho, z)$ can be used as global coordinates on the interior of $\hat{M}$, so we may identify the interior of the orbit space with the upper-half plane

$$
\hat{M}=\{(\rho, z) \mid \rho>0\}
$$

\footnotetext{
5 This is equivalent to the Frobenius integrability condition $K_{0} \wedge K_{1} \wedge K_{2} \wedge \mathrm{d} K_{A}=0$, which is in fact guaranteed for any solution to the Einstein-Maxwell equation with $D-2$ commuting Killing fields, one of which has at least one fixed point (which must be the case here due to asymptotic flatness), see e.g. [44]. Here it arises as a consequence of supersymmetry which for timelike solutions implies the Einstein-Maxwell equations [36].
} 
and the boundary $\partial \hat{M}$ and corners with the $z$-axis $(\rho=0)$. In the orbit space the axes divide into boundary segments $I=\left(z_{1}, z_{2}\right)$, called axis rods (or intervals), and corners which arise as certain endpoints $z=z_{i}$ of the axis rods. Below we will show that in the orbit space an event horizon is a point on the $z$-axis.

Since det $G_{A B}<0$ on $\langle\langle M\rangle\rangle \backslash \mathcal{A}$, the orbit space metric (36) must be Riemannian on $\hat{M}$ and therefore its conformal factor must be a smooth and positive function for $\rho>0$, i.e.

$$
\frac{H}{f}>0 \text {. }
$$

In fact, from (17), we see that (43) is equivalent to the invariant $N>0$. Thus the above shows that $N>0$ on $\langle\langle M\rangle\rangle \backslash \mathcal{A}$. Therefore, Lemma 1 may be applied to learn that the harmonic functions $H, K, L, M$ are smooth on $\langle\langle M\rangle\rangle \backslash \mathcal{A}$.

We require that the spacetime metric is smooth at the axes. Consider an axis rod $I$ and let $v=v_{i} m_{i}$, where $\left(v_{1}, v_{2}\right) \in \mathbb{Z}^{2}$, denote the Killing field which vanishes on $I$. Smoothness requires that for each such axis $\operatorname{rod} I$

$$
\frac{H}{f}=\lim _{\rho \rightarrow 0} \frac{\gamma_{i j} v_{i} v_{j}}{\rho^{2}}
$$

is a smooth positive function for all $z \in I{ }^{6}$ To see this, consider the spacetime metric for fixed $z \in I$,

$$
\mathrm{d} s^{2}=\frac{H}{f} \mathrm{~d} \rho^{2}+G_{t t} \mathrm{~d} t^{2}+2 G_{t i} \mathrm{~d} t \mathrm{~d} \phi^{i}+G_{i j} \mathrm{~d} \phi^{i} \mathrm{~d} \phi^{j} .
$$

By an $S L(2, \mathbb{Z})$ transformation we may assume that $v=m_{1}=\partial_{\phi^{1}}=0$ on $I$, so $G_{A 1}=0$ on $I$ for all $A$. The metric is smooth on $I$ provided $\left(\mathrm{d}\left|m_{1}\right|\right)^{2} \rightarrow 1$ as $\rho \rightarrow 0$. In terms of the proper distance $s=\int_{0}^{\rho} \sqrt{g_{\rho \rho}} \mathrm{d} \rho$ this implies $G_{11}=s^{2}+O\left(s^{4}\right)$ and $G_{1 t}=G_{1 i}=O\left(s^{2}\right)$. Therefore

$$
-\rho^{2}=\operatorname{det} G=\left|\begin{array}{ll}
G_{t t} & G_{t 2} \\
G_{2 t} & G_{22}
\end{array}\right| s^{2}+O\left(s^{4}\right) .
$$

Now, it has been shown that $\operatorname{span}\left\{K_{0}, K_{1}, K_{2}\right\}$ is timelike everywhere in $\langle\langle M\rangle\rangle[41]$. Therefore, $\operatorname{span}\left\{V, m_{2}\right\}$ must be timelike on $I$ and hence the determinant on the righthand side of (46) is strictly negative on $I$. Thus,

$$
\frac{f}{H}=\left(\frac{\mathrm{d} \rho}{\mathrm{d} s}\right)^{2}=-\left|\begin{array}{ll}
G_{t t} & G_{t 2} \\
G_{2 t} & G_{22}
\end{array}\right|+O\left(s^{2}\right)
$$

is smooth and positive on $I$. The condition (44) now easily follows. From (17) we deduce that the invariant $N>0$ on all axis rods.

Thus we arrive at the following crucial result.

Lemma 3. Let $(M, g, F)$ be an asymptotically flat, supersymmetric and biaxisymmetric solution to minimal supergravity with a globally hyperbolic $\langle\langle M\rangle\rangle$.

1. The fixed points of the triholomorphic Killing field of the Gibbons-Hawking base correspond to precisely the corners of the orbit space $\hat{M}$.

\footnotetext{
6 This condition has been previously derived for vacuum Weyl solutions [10,19,21].
} 
2. The harmonic functions $H, K, L, M$ are smooth and obey (23) everywhere in $\langle\langle M\rangle\rangle$ except possibly at points corresponding to the corners of the orbit space $\hat{M}$.

3. At every corner of the orbit space $f \neq 0$ and $H$ has an isolated singularity.

Proof. The fixed points of $\partial_{\psi}$ are on $\mathcal{A}$ and therefore must either occur on an axis rod or on a corner of $\hat{M}$. But $\partial_{\psi}=0$ implies that the invariant $N=0$ (recall (16)), which as we have shown above cannot occur on an axis rod. Therefore, $\partial_{\psi}=0$ can only occur at a corner of $\hat{M}$. Conversely, by definition, at any corner $m_{1}=m_{2}=0$ and hence $\partial_{\psi}=0$. Thus we have proved part 1.

Next, above we observed that the invariant $N>0$ on $\langle\langle M\rangle\rangle \backslash \mathcal{A}$ and also on the parts of $\mathcal{A}$ corresponding to the axis rods. Thus by application of Lemma 1 we deduce that $H, K, L, M$ are smooth and obey (23) on $\langle\langle M\rangle\rangle \backslash \mathcal{A}$ and also on the parts of $\mathcal{A}$ corresponding to the axis rods. Thus the only potential singularities of the harmonic functions in $\langle\langle M\rangle\rangle$ are points corresponding to the corners of the orbit space, establishing 2.

Finally, we again use the fact that $\operatorname{span}\left\{K_{0}, K_{1}, K_{2}\right\}$ is timelike everywhere in $\langle\langle M\rangle\rangle$ [41]. In particular, this implies that $K_{0}=V$ must be timelike at any corner of $\hat{M}$. Therefore, $f \neq 0$ at any corner. Also, as observed above $N=0$ at the corners. Therefore, the expression for $H$ in terms of these invariants (17) shows that $H$ must be singular at the corners of $\hat{M}$. Since we have already established that $H$ is smooth on the orbit space away from the corners and horizon, we deduce the singularities of $H$ are isolated.

Therefore the analysis reduces to studying the behaviour of the harmonic functions at the event horizon and the corners the of orbit space.

\section{Near-Horizon Geometry}

We will now examine the geometry near a horizon in detail. The event horizon $\mathcal{H}$ of a black hole must be invariant under the isometries of the spacetime. Hence any Killing field must be tangent to $\mathcal{H}$, which implies it must be null or spacelike on $\mathcal{H}$. In particular, the supersymmetric Killing field $V$ must be tangent and hence null on $\mathcal{H}$ (since it is never spacelike). Hence $V$ is also normal to the horizon and $\mathcal{H}$ is a Killing horizon with respect to $V$. Furthermore, $\mathrm{d}(V \cdot V)=0$ on $\mathcal{H}$, so the horizon must be degenerate (i.e. extreme). We will refer to such horizons as supersymmetric horizons. Since for asymptotically flat solutions $V$ is also the stationary Killing field we deduce that any supersymmetric black hole is nonrotating.

In the neighbourhood of a supersymmetric horizon we may introduce Gaussian null coordinates $\left(v, \lambda, x^{a}\right)[46]$

$$
\mathrm{d} s^{2}=-\lambda^{2} \Delta(\lambda, x)^{2} \mathrm{~d} v^{2}+2 \mathrm{~d} v \mathrm{~d} \lambda+2 \lambda h_{a}(\lambda, x) \mathrm{d} v \mathrm{~d} x^{a}+\gamma_{a b}(\lambda, x) \mathrm{d} x^{a} \mathrm{~d} x^{b},
$$

where $V=\partial_{v}, \lambda=0$ is the horizon and $f=\lambda \Delta(\lambda, x)$. The supersymmetric nearhorizon geometries of minimal supergravity have been classified [32]. Assuming crosssections of the horizon are compact, it can be shown that $\left.\Delta\right|_{\lambda=0}=\Delta_{0}$ is a constant on the horizon. If $\Delta_{0} \neq 0$ the horizon is locally $S^{3}$, and if $\Delta_{0}=0$ it is $S^{1} \times S^{2}$ (we do not consider the $T^{3}$ case as this is not an allowed topology for black holes). ${ }^{7}$ In fact, an

\footnotetext{
7 The analysis of [32] assumes $f>0$ (i.e. $\Delta>0$ ) for small $r>0$. However, this is restrictive and we should only assume $f \neq 0$ (i.e. $\Delta \neq 0$ for small $r>0$ ). In fact, the analysis of [32] remains valid under this weaker assumption since only $\Delta^{2}$ appears in the near-horizon geometry.
} 
output of this analysis is that the near-horizon geometry must have biaxial symmetry. We will use this classification below after a general analysis of the orbit space.

3.1. Orbit space metric. Let us now consider the orbit space metric near an extreme horizon. Our analysis in this section will be general and only assume the existence of biaxial symmetry.

The biaxial Killing fields must be tangent to the horizon and hence on a cross-section we may introduce coordinates adapted to this symmetry. We thus introduce Gaussian null coordinates $\left(v, \lambda, \tilde{\theta}, \tilde{\phi}^{i}\right)$ where $\left(\tilde{\theta}, \tilde{\phi}^{i}\right), i=1,2$, are such that the three commuting Killing fields are $V=\partial_{v}, m_{i}=\partial_{\tilde{\phi}^{i}}$. Then we write

$$
\begin{aligned}
\mathrm{d} s^{2}= & -\lambda^{2} F \mathrm{~d} v^{2}+2 \mathrm{~d} v\left(\mathrm{~d} \lambda+\lambda \tilde{h}_{\tilde{\theta}} \mathrm{d} \tilde{\theta}\right)+\gamma_{\tilde{\theta} \tilde{\theta}} \mathrm{d} \tilde{\theta}^{2}+2 \gamma_{\tilde{\theta} i} \mathrm{~d} \tilde{\theta}\left(\mathrm{d} \tilde{\phi}^{i}+\lambda h^{i} \mathrm{~d} v\right) \\
& +\gamma_{i j}\left(\mathrm{~d} \tilde{\phi}^{i}+\lambda h^{i} \mathrm{~d} v\right)\left(\mathrm{d} \tilde{\phi}^{j}+\lambda h^{j} \mathrm{~d} v\right),
\end{aligned}
$$

where $F=\Delta^{2}+h^{i} h_{i}, \tilde{h}_{\tilde{\theta}}=h_{\tilde{\theta}}-\gamma_{\tilde{\theta} i} h^{i}$ and $h^{i}=\gamma^{i j} h_{j}$ where $\gamma^{i j}$ is the inverse of the $2 \mathrm{~d}$ matrix $\gamma_{i j}$. We will now extract the orbit space metric, following [47].

The inner product on the space of Killing fields is

$$
G=-\lambda^{2} F \mathrm{~d} v^{2}+\gamma_{i j}\left(\mathrm{~d} \tilde{\phi}^{i}+\lambda h^{i} \mathrm{~d} v\right)\left(\mathrm{d} \tilde{\phi}^{j}+\lambda h^{j} \mathrm{~d} v\right)
$$

with inverse

$$
G^{A B}=\left(\begin{array}{cc}
-\frac{1}{\lambda^{2} F} & \frac{h^{i}}{\lambda F} \\
\frac{h^{j}}{\lambda F} & \gamma^{i j}-\frac{h^{i} h^{j}}{F}
\end{array}\right) .
$$

The orbit space metric may be defined by

$$
q_{\mu \nu}=g_{\mu \nu}-G^{A B} g_{A \mu} g_{B \nu}
$$

so $q_{A v}=0$. Computing we find

$$
q=\frac{1}{F \lambda^{2}}\left(\mathrm{~d} \lambda+\lambda \tilde{h}_{\tilde{\theta}} \mathrm{d} \tilde{\theta}\right)^{2}+\left(\gamma_{\tilde{\theta} \tilde{\theta}}-\gamma^{i j} \gamma_{i \tilde{\theta}} \gamma_{j \tilde{\theta}}\right) \mathrm{d} \tilde{\theta}^{2}
$$

An alternate way to derive this orbit space metric is as follows.

The spacetime has three commuting Killing fields. Hence the distribution orthogonal to these Killing fields is integrable and there exist local coordinates in which the metric takes block diagonal form as (36) (see e.g. [44]). The general coordinate change which takes us from Gaussian null coordinates to block diagonal coordinates is

$$
t=v+A(\lambda, \tilde{\theta}), \quad \phi^{i}=\tilde{\phi}^{i}+B^{i}(\lambda, \tilde{\theta}),
$$

where $V=\partial_{t}, m_{i}=\partial_{\phi^{i}}$ and

$$
\partial_{\lambda} A=-\frac{1}{\lambda^{2} F}, \quad \partial_{\tilde{\theta}} A=-\frac{\tilde{h}_{\tilde{\theta}}}{\lambda F}, \quad \partial_{\lambda} B^{i}=\frac{h^{i}}{\lambda F}, \quad \partial_{\tilde{\theta}} B^{i}=\gamma^{i j} \gamma_{\tilde{\theta} j}+\frac{h^{i} \tilde{h}_{\tilde{\theta}}}{F} .
$$

A calculation then shows that the spacetime metric takes the form (34) with the matrix of Killing fields

$$
G=-\lambda^{2} F \mathrm{~d} t^{2}+\gamma_{i j}\left(\mathrm{~d} \phi^{i}+\lambda h^{i} \mathrm{~d} t\right)\left(\mathrm{d} \phi^{j}+\lambda h^{j} \mathrm{~d} t\right)
$$


and the orbit space metric given by (53).

We will now examine the orbit space metric near the horizon. We will assume $h_{\tilde{\theta}}=$ $O(\lambda)$ and $\gamma_{i \tilde{\theta}}=O(\lambda)$ which are conditions satisfied by the near-horizon geometries in question. We find that near the horizon (53) takes the form

$$
q=\left(\frac{1}{F \lambda^{2}}+O\left(\lambda^{-1}\right)\right) \mathrm{d} \lambda^{2}+O(1) \mathrm{d} \lambda \mathrm{d} \tilde{\theta}+\left(\gamma_{\tilde{\theta} \tilde{\theta}}+O(\lambda)\right) \mathrm{d} \tilde{\theta}
$$

and the determinant of the Killing metric is

$$
\begin{aligned}
\rho & =\sqrt{-\operatorname{det} G}=\lambda \sqrt{F \operatorname{det} \gamma_{i j}} \\
& =\left.\sqrt{F \operatorname{det} \gamma_{i j}}\right|_{\lambda=0} \lambda+O\left(\lambda^{2}\right) .
\end{aligned}
$$

The function $\rho$ is harmonic in the orbit space metric (36). The harmonic conjugate $z$ is given by $\mathrm{d} z=\star_{2} \mathrm{~d} \rho$. A computation gives

$$
\begin{aligned}
\partial_{\lambda} z & =\frac{1}{\lambda \sqrt{F q_{\tilde{\theta} \tilde{\theta}}}}\left(\partial_{\tilde{\theta}} \rho-\lambda \tilde{h}_{\tilde{\theta}} \partial_{\lambda} \rho\right)=\left.\frac{\partial_{\tilde{\theta}} \sqrt{F \operatorname{det} \gamma_{i j}}}{\sqrt{F \gamma_{\tilde{\theta} \tilde{\theta}}}}\right|_{\lambda=0}+O(\lambda), \\
\partial_{\tilde{\theta}} z & =\frac{1}{\sqrt{F q_{\tilde{\theta} \tilde{\theta}}}}\left[-\lambda F q_{\tilde{\theta} \tilde{\theta}} \partial_{\lambda} \rho+\tilde{h}_{\tilde{\theta}}\left(\partial_{\tilde{\theta}} \rho-\lambda \tilde{h}_{\tilde{\theta}} \partial_{\lambda} \rho\right)\right] \\
& =-\left.F \sqrt{\gamma_{\tilde{\theta} \tilde{\theta}} \operatorname{det} \gamma_{i j}}\right|_{\lambda=0} \lambda+O\left(\lambda^{2}\right) .
\end{aligned}
$$

Observe that by integrating for $z$ we see that it will take the form $z=z_{0}+O(\lambda)$, where $z_{0}$ is a constant which can be set to zero. Hence, a degenerate horizon corresponds to a single point on the boundary of the orbit space. In fact, in the orbit space metric, it is easy to see that any point is an infinite proper distance to the horizon, so that a degenerate horizon corresponds to an asymptotic end (see [45] for discussion of this in the vacuum case).

To proceed further we need the specific near-horizon geometries. We will turn to this next.

3.2. Locally $S^{3}$ horizon. For a locally $S^{3}$ horizon, the horizon data is given by [32]

$$
\begin{aligned}
\Delta_{0}^{2} & =\frac{4}{\mu}\left(1-\frac{j^{2}}{\mu^{3}}\right),\left.\quad h\right|_{\lambda=0}=-j \mu^{-3 / 2}\left(1-\frac{j^{2}}{\mu^{3}}\right)^{1 / 2}(\mathrm{~d} \tilde{\psi}+\cos \tilde{\theta} \mathrm{d} \tilde{\phi}), \\
\left.\gamma\right|_{\lambda=0} & =\frac{\mu}{4}\left[\left(1-\frac{j^{2}}{\mu^{3}}\right)(\mathrm{d} \tilde{\psi}+\cos \tilde{\theta} \mathrm{d} \tilde{\phi})^{2}+\mathrm{d} \tilde{\theta}^{2}+\sin ^{2} \tilde{\theta} \mathrm{d} \tilde{\phi}^{2}\right],
\end{aligned}
$$

where the constants $j^{2}<\mu^{3}$. We deduce that near the horizon the metric is given by (49) where

$$
\begin{aligned}
F & =\frac{4}{\mu}+O(\lambda), \quad h^{i}=-\frac{4 j}{\mu^{5 / 2}\left(1-\frac{j^{2}}{\mu^{3}}\right)^{1 / 2}} \delta_{\tilde{\psi}}^{i}+O(\lambda), \\
h_{\tilde{\theta}} & =O(\lambda), \quad \gamma_{\tilde{\theta} \tilde{\theta}}=\frac{\mu}{4}+O(\lambda), \quad \gamma_{\tilde{\theta} i}=O(\lambda), \\
\gamma_{i j} \mathrm{~d} \tilde{\phi}^{i} \mathrm{~d} \tilde{\phi}^{j} & =\frac{\mu}{4}\left[\left(1-\frac{j^{2}}{\mu^{3}}\right)(\mathrm{d} \tilde{\psi}+\cos \tilde{\theta} \mathrm{d} \tilde{\phi})^{2}+\sin ^{2} \tilde{\theta} \mathrm{d} \tilde{\phi}^{2}\right]+O(\lambda) .
\end{aligned}
$$


The above horizon geometry is locally that of a squashed $S^{3}$ with a $U(1)^{2}$-isometry generated by the Killing fields $\left(\partial_{\tilde{\psi}}, \partial_{\tilde{\phi}}\right)$. The topology of the horizon is determined by the periodicity lattice of the biaxial Killing fields. For now our analysis will be local, but we will consider global constraints at the end of this section. In any case, locally, biaxial symmetry implies that $\left(\partial_{\tilde{\psi}}, \partial_{\tilde{\phi}}\right)$ must be related to the GH space biaxial Killing fields $\left(\partial_{\psi}, \partial_{\phi}\right)$ by a constant linear transformation. Hence, $\left.\operatorname{det} \gamma_{i j}\right|_{i, j=\tilde{\psi}, \tilde{\phi}}=\left.c^{2} \operatorname{det} \gamma_{i j}\right|_{i, j=\psi, \phi}$ for some constant $c>0$. We need to take account of this Jacobian when comparing the determinants of the matrix of Killing fields to determine the Weyl coordinates $(\rho, z)$.

Using the near horizon data (61), we find that (58) and (59) imply

$$
\rho=\sqrt{\frac{\mu}{4 c^{2}}\left(1-\frac{j^{2}}{\mu^{3}}\right)} \lambda \sin \tilde{\theta}+O\left(\lambda^{2}\right), \quad z=\sqrt{\frac{\mu}{4 c^{2}}\left(1-\frac{j^{2}}{\mu^{3}}\right)} \lambda \cos \tilde{\theta}+O\left(\lambda^{2}\right) .
$$

Thus the $\mathbb{R}^{3}$ polar coordinates are

$$
r=\sqrt{\frac{\mu}{4 c^{2}}\left(1-\frac{j^{2}}{\mu^{3}}\right)} \lambda+O\left(\lambda^{2}\right), \quad \cos \theta=\cos \tilde{\theta}+O(\lambda) .
$$

We deduce that the horizon is a single point in the orbit space metric, as anticipated above. The orbit space metric in the $(\rho, z)$ coordinates must take the form (36), so using the coordinate change (62) we find

$$
q=\frac{H}{f}\left[\frac{\mu}{4 c^{2}}\left(1-\frac{j^{2}}{\mu^{3}}\right)\left(\mathrm{d} \lambda^{2}+\lambda^{2} \mathrm{~d} \tilde{\theta}^{2}\right)+O(\lambda) \mathrm{d} \lambda^{2}+O\left(\lambda^{2}\right) \mathrm{d} \lambda \mathrm{d} \tilde{\theta}+O\left(\lambda^{3}\right) \mathrm{d} \tilde{\theta}^{2}\right]
$$

and comparing to (57) implies

$$
\frac{H}{f}=\frac{c^{2}}{1-\frac{j^{2}}{\mu^{3}}}\left(\frac{1}{\lambda^{2}}+O\left(\lambda^{-1}\right)\right)
$$

Therefore, using $f=\lambda \Delta_{0}+O\left(\lambda^{2}\right)$ we deduce

$$
H=\frac{4 c^{2}}{\mu \Delta_{0} \lambda}+O(1)=\frac{h_{0}}{r}+O(1)
$$

where we have defined the constant $h_{0}=\operatorname{sgn}\left(\Delta_{0}\right) c$. Observe that the first term in $H$ is harmonic and hence the $O(1)$ term is also a harmonic function. Therefore, we deduce that a horizon corresponds to an isolated singularity of the harmonic function $H$; specifically the horizon is a pole of order one.

We now turn to the remaining harmonic functions. Firstly, observe that we can write $\partial_{\psi}=a \partial_{\tilde{\psi}}+b \partial_{\tilde{\phi}}$ for some constants $a, b$. Hence the invariant $g_{t \psi}=V \cdot \partial_{\psi}=\lambda h \cdot \partial_{\psi}=$ $\lambda\left(a h_{\tilde{\psi}}+b h_{\tilde{\phi}}\right)$. Then, the near-horizon expansion of the invariants $g_{t \psi}$ and $f$, together with (18) and smoothness of $\Psi$, imply that $K H^{-1}$ is smooth at $\lambda=0$. Therefore $K=O\left(r^{-1}\right)$ and since it is harmonic it must have a pole of order one, so

$$
K=\frac{k_{0}}{r}+O(1),
$$

where $k_{0}$ is a constant and the $O(1)$ term is harmonic. Due to the shift freedom in $K$ (21) the constant $k_{0}$ can be set to any value. 
Next, using the expansion of the invariants $f$ and $-f^{2} \omega_{\psi}=V \cdot \partial_{\psi}$ together with (4) and (6) implies

$$
L=\frac{\ell_{0}}{r}+O(1), \quad M=\frac{1}{r}\left(\frac{j(a+b \cos \theta)}{8 c}+\frac{k_{0}^{3}}{2 h_{0}^{2}}-\frac{3 \mu k_{0}}{8 h_{0}^{2}}\right)+O(1),
$$

where $\ell_{0}=h_{0}^{-1}\left(\frac{1}{4} \mu-k_{0}^{2}\right)$ and hence $L$ has a pole of order one. Also we have $M=$ $O\left(r^{-1}\right)$ and since it is harmonic this implies $M$ also has a pole of order one so must be of the form $M=m_{0} / r+O(1)$ where $m_{0}$ is a constant. Hence $b=0$ so we deduce the triholomorphic Killing field $\partial_{\psi}=a \partial_{\tilde{\psi}}$. In summary, so far we have shown that a regular horizon corresponds to a simple pole of the harmonic functions $H, K, L, M$.

We now turn to global constraints. The precise periodicities of $(\tilde{\psi}, \tilde{\phi})$ determine the horizon topology which in general may be that of a lens space. Now, asymptotic flatness fixes the identifications of the GH space angles $(\psi, \phi)$ to be standard Euler angles on $S^{3}$ (30). This will impose identifications on the $(\tilde{\psi}, \tilde{\phi})$ angles. To analyse this, it is convenient to note that the Killing vectors on the horizon which have fixed points at the poles $\tilde{\theta}=0, \pi$ are $\tilde{v}_{ \pm}=\partial_{\tilde{\phi}} \mp \partial_{\tilde{\psi}}$. For a lens space $L(p, q)$ these must be related to the independently $2 \pi$-periodic vectors (30) of the asymptotically flat region by

$$
\left(\begin{array}{c}
\tilde{v}_{-} \\
\tilde{v}_{+}
\end{array}\right)=A\left(\begin{array}{c}
v_{-} \\
v_{+}
\end{array}\right)
$$

where $A \in G L(2, \mathbb{Z})$ and $\operatorname{det} A=p \in \mathbb{Z}$. The corresponding transformation in terms of Euler angles can be deduced from (30), which implies $\left.\operatorname{det} \gamma_{i j}\right|_{i, j=\tilde{\psi}, \tilde{\phi}}=$ $\left.p^{2} \operatorname{det} \gamma_{i j}\right|_{i, j=\psi, \phi}$. Thus, comparing to our local analysis above shows that $c= \pm p$ is precisely the integer which defines the lens spaces $L(p, q)$. In fact, by fixing the sign of $p$ appropriately we will identify the constant in (66) as

$$
h_{0}=p
$$

Equations (66)-(68) derived in this section are necessary conditions for regularity at the horizon. We will examine sufficient conditions for a regular horizon in Sect. 3.4.

3.3. $S^{1} \times S^{2}$ horizon. We will now repeat the above analysis for the other type of near-horizon geometry. The horizon data is now

$$
\begin{aligned}
\Delta_{0} & =0,\left.\quad h\right|_{\lambda=0}=\frac{R}{\ell} \mathrm{d} \tilde{\psi}, \\
\left.\gamma\right|_{\lambda=0} & =R^{2} \mathrm{~d} \tilde{\psi}^{2}+\ell^{2}\left(\mathrm{~d} \tilde{\theta}^{2}+\sin ^{2} \tilde{\theta} \mathrm{d} \tilde{\phi}^{2}\right),
\end{aligned}
$$

where the constant $R>0$ has been introduced for later convenience. Thus, near the horizon the metric is given by (49) where

$$
\begin{aligned}
F & =\frac{1}{\ell^{2}}+O(\lambda), \quad h^{i}=\frac{1}{R \ell} \delta_{\tilde{\psi}}^{i}+O(\lambda), \\
h_{\tilde{\theta}} & =O(\lambda), \quad \gamma_{\tilde{\theta} \tilde{\theta}}=\ell^{2}+O(\lambda), \quad \gamma_{\tilde{\theta} i}=O(\lambda), \\
\gamma_{i j} \mathrm{~d} \tilde{\phi}^{i} \mathrm{~d} \tilde{\phi}^{j} & =R^{2} \mathrm{~d} \tilde{\psi}^{2}+\ell^{2} \sin ^{2} \tilde{\theta} \mathrm{d} \tilde{\phi}^{2}+O(\lambda) .
\end{aligned}
$$


The near-horizon geometry has biaxial symmetry generated by the Killing fields $\partial_{\tilde{\psi}}, \partial_{\tilde{\phi}}$. As before, these must be related by a constant linear transformation to the biaxial Killing fields of the GH space, so det $\left.\gamma_{i j}\right|_{i, j=\tilde{\psi}, \tilde{\phi}}=\left.c^{2} \operatorname{det} \gamma_{i j}\right|_{i, j=\psi, \phi}$ for some constant $c>0$. Then, using (58) and (59) we find

$$
\rho=\frac{R}{c} \lambda \sin \tilde{\theta}+O\left(\lambda^{2}\right), \quad z=\frac{R}{c} \lambda \cos \tilde{\theta}+O\left(\lambda^{2}\right),
$$

so the $\mathbb{R}^{3}$ polar coordinates are

$$
r=\frac{R}{c} \lambda+O\left(\lambda^{2}\right), \quad \cos \theta=\cos \tilde{\theta}+O(\lambda)
$$

Hence, again, the horizon corresponds to a point in the orbit space metric.

We may now compare to the orbit space metric (36) and (57) near the horizon. Using (73) we find

$$
\frac{H}{f}=\frac{c^{2} \ell^{2}}{R^{2} \lambda^{2}}+O\left(\lambda^{-1}\right)=\frac{\ell^{2}}{r^{2}}+O\left(r^{-1}\right) .
$$

The function $f=\lambda^{2} \tilde{\Delta}+O\left(\lambda^{3}\right)$, for some non-zero constant $\tilde{\Delta}$ [32]. Thus we learn that

$$
H=\frac{\tilde{\Delta} \ell^{2}}{R^{2}}+O(\lambda)
$$

so in this case $H$ is a smooth harmonic function at the horizon.

We now determine the other harmonic functions. Near the horizon the invariant $g_{t \psi}=V \cdot \partial_{\psi}=\lambda h \cdot \partial_{\psi}=\lambda a h_{\tilde{\psi}}+O\left(\lambda^{2}\right)$, where the final equality follows from writing $\partial_{\psi}=a \partial_{\tilde{\psi}}+b \partial_{\tilde{\phi}}$ for constants $a, b$. Then, expanding (18) near the horizon we find

$$
K H^{-1}=-\frac{a R}{\ell \tilde{\Delta} \lambda}+O(1)
$$

where we have used the near-horizon expansion of $f$ and smoothness of $\Psi$. Hence

$$
K=-\frac{a \ell}{R \lambda}+O(1)=-\frac{a \ell}{c r}+O(1) .
$$

Using the invariants $f$ and $V \cdot \partial_{\psi}=-f^{2} \omega_{\psi}$, together with (4) and (6), then implies

$$
L=\frac{\left(1-a^{2}\right) R^{2}}{\tilde{\Delta} c^{2} r^{2}}+O\left(r^{-1}\right), \quad M=\frac{a\left(1-a^{2}\right) R^{4}}{\tilde{\Delta} \ell c^{3} r^{3}}+O\left(r^{-2}\right) .
$$

Therefore $L$ has a pole of order at most two. However, a harmonic function in $\mathbb{R}^{3}$ with a pole of order two must be of the form $L=c_{1} r^{-2} \cos \theta+c_{2} r^{-1}+O(1)$. Thus, since the coefficient of the $r^{-2}$ term is a constant it must vanish and hence we have $a=1$ (choosing a sign). Hence $L=O\left(r^{-1}\right)$ so harmonicity implies it has a pole of at most order one. This then also implies $M$ has a pole of at most order two.

In fact we may show that $M=O\left(r^{-1}\right)$ as follows. The explicit expression for the invariant $g_{\psi \psi}$ in (10), together the above behaviour of the harmonic functions requires $K^{3} M /\left(H L+K^{2}\right)^{2}$ to be smooth at $\lambda=0$. It follows that

$$
M=O\left(r^{-1}\right)
$$


and hence harmonicity implies it must also have a pole of order one.

We now turn to global constraints imposed by asymptotic flatness. In the case of a $S^{1} \times S^{2}$ horizon, $\partial_{\tilde{\psi}}$ and $\partial_{\tilde{\phi}}$ are independently periodic and we will choose the constant $R$ so that $\tilde{\psi}$ has period $4 \pi$. Hence we can relate $2 \partial_{\tilde{\psi}}$ and $\partial_{\tilde{\phi}}$ to the independently $2 \pi$ periodic vectors $v_{+}, v_{-}$of the asymptotically flat region $(30)$ by a $S L(2, \mathbb{Z})$ transformation. Their relation to the Euler angles of the Gibbons-Hawking base can then be deduced from (30). We find det $\left.\gamma_{i j}\right|_{i, j=\tilde{\psi}, \tilde{\phi}}=\left.\operatorname{det} \gamma_{i j}\right|_{i, j=\psi, \phi}$ so we deduce the constant $c=1$.

We will examine sufficient conditions for regularity of the horizon in Sect. 3.4.

3.4. Horizon regularity and topology. As we have seen above a regular horizon corresponds to an isolated singularity in the $\mathbb{R}^{3}$ base of the GH space which we may take to be the origin $r=0$. Furthermore, the harmonic functions have at most simple poles at the horizon and so can be written as

$$
H=\frac{h_{0}}{r}+H_{0}, \quad K=\frac{k_{0}}{r}+K_{0}, \quad L=\frac{\ell_{0}}{r}+L_{0}, \quad M=\frac{m_{0}}{r}+M_{0},
$$

where $h_{0}, k_{0}, \ell_{0}, m_{0}$ are constants and $H_{0}, K_{0}, L_{0}, M_{0}$ are harmonic functions that are smooth at $r=0$. Thus we can write $H_{0}=c_{0}+O(r)$, where $c_{0}$ is a constant and the $O(r)$ term is analytic in $r$, and similarly for the other harmonic functions. In particular, using (10) this implies

$$
\begin{aligned}
\frac{H}{f} & =\frac{\alpha_{0}}{r^{2}}+\frac{\alpha_{1}}{r}+O(1), \\
g_{\psi \psi} & =\beta_{0}+r \beta_{1}+O\left(r^{2}\right), \\
g_{t \psi} & =r\left(\gamma_{0}+r \gamma_{1}+O\left(r^{2}\right)\right),
\end{aligned}
$$

where $\alpha_{i}, \beta_{i}, \gamma_{i}$ are constants and comparing to the near-horizon analysis in the previous sections implies

$$
\alpha_{0}>0, \quad \beta_{0}>0 \text {. }
$$

Then we can write

$$
f=r\left(\frac{h_{0}}{\alpha_{0}}+\frac{c_{0} \alpha_{0}-h_{0} \alpha_{1}}{\alpha_{0}^{2}} r+O\left(r^{2}\right)\right) .
$$

The explicit expressions for leading order coefficients are

$$
\begin{aligned}
\alpha_{0} & =h_{0} \ell_{0}+k_{0}^{2}, \\
\beta_{0} & =\frac{-h_{0}^{2} m_{0}^{2}-3 h_{0} k_{0} \ell_{0} m_{0}+h_{0} \ell_{0}^{3}-2 k_{0}^{3} m_{0}+\frac{3}{4} k_{0}^{2} \ell_{0}^{2}}{\alpha_{0}^{2}}, \\
\gamma_{0}^{2} & =\frac{\alpha_{0}-h_{0}^{2} \beta_{0}}{\alpha_{0}^{2}}=\left(\frac{h_{0}^{2} m_{0}+\frac{3}{2} h_{0} k_{0} \ell_{0}+k_{0}^{3}}{\alpha_{0}^{2}}\right)^{2} .
\end{aligned}
$$

Notice that the last relation shows that $\gamma_{0}^{2} \geq 0$ does not lead to any inequalities beyond (83). In fact, from the very same relation we can see that $\alpha_{0}^{2} \beta_{0}>0$ actually implies $\alpha_{0}>0$ so (83) is really equivalent to the single condition

$$
-h_{0}^{2} m_{0}^{2}-3 h_{0} k_{0} \ell_{0} m_{0}+h_{0} \ell_{0}^{3}-2 k_{0}^{3} m_{0}+\frac{3}{4} k_{0}^{2} \ell_{0}^{2}>0
$$


on the parameters $h_{0}, k_{0}, \ell_{0}, m_{0}$. It is worth noting that

$$
K^{2}+H L=\frac{\alpha_{0}}{r^{2}}+O\left(r^{-1}\right), \quad g^{t t}=-\frac{\alpha_{0} \beta_{0}}{r^{2}}+O\left(r^{-1}\right)
$$

which already confirms that the above inequalities imply the solution is smooth (23) and stably causal (25) near (but not at) the horizon. We will now show that (86) is sufficient for regularity at the horizon.

To this end, let us perform a coordinate transformation

$$
\mathrm{d} t=\mathrm{d} v+\left(\frac{A_{0}}{r^{2}}+\frac{A_{1}}{r}\right) \mathrm{d} r, \quad \mathrm{~d} \psi=\mathrm{d} \psi^{\prime}+\frac{B_{0}}{r} \mathrm{~d} r+C \mathrm{~d} \phi^{\prime}, \quad \mathrm{d} \phi=\mathrm{d} \phi^{\prime},
$$

where $A_{0}, A_{1}, B_{0}, C$ are constants to be determined. Using the above expansions, it follows that $g_{r r}$ contains $1 / r^{2}$ and $1 / r$ singular terms, whereas $g_{r} \psi^{\prime}$ contains $1 / r$ singular terms. Demanding that the $1 / r^{2}$ term in $g_{r r}$ and $1 / r$ term in $g_{t \psi^{\prime}}$ vanish is equivalent to setting

$$
A_{0}^{2}=\beta_{0} \alpha_{0}^{2}, \quad B_{0}=-\frac{A_{0} \gamma_{0}}{\beta_{0}} .
$$

Demanding that the $1 / r$ term in $g_{r r}$ vanishes fixes

$$
A_{1}=\frac{\alpha_{0} \beta_{0}}{2 A_{0}}\left(B_{0}^{2} \beta_{1}+2 B_{0} A_{0} \gamma_{1}+\alpha_{1}-\frac{2 h_{0}\left(c_{0} \alpha_{0}-h_{0} \alpha_{1}\right)}{\alpha_{0}^{3}} A_{0}^{2}\right) .
$$

Note that we have simplified $A_{0}, A_{1}$ using the identity for $\gamma_{0}$ above. With these choices, $g_{r r}$ and $g_{r} \psi^{\prime}$ are analytic at $r=0$.

We will also need the near-horizon behaviour of the 1 -forms $\chi, \hat{\omega}, \xi$. Using the behaviour of the harmonic functions (81) near the horizon we find

$$
\begin{aligned}
& \star_{3} \mathrm{~d} \chi=\left(-h_{0} r^{-2}+O(1)\right) \mathrm{d} r+O(r) \mathrm{d} \theta, \\
& \star_{3} \mathrm{~d} \hat{\omega}=O\left(r^{-2}\right) \mathrm{d} r+O(1) \mathrm{d} \theta, \\
& \star_{3} \mathrm{~d} \xi=\left(k_{0} r^{-2}+O(1)\right) \mathrm{d} r+O(r) \mathrm{d} \theta,
\end{aligned}
$$

and writing the 1-forms as (33), we may integrate to get

$$
\chi=\left(h_{0} \cos \theta+\tilde{\chi}_{0}+O\left(r^{2}\right)\right) \mathrm{d} \phi, \quad \hat{\omega}=O(1) \mathrm{d} \phi, \quad \xi=\left(-k_{0} \cos \theta+\tilde{\xi}_{0}+O\left(r^{2}\right)\right) \mathrm{d} \phi
$$

for some constants $\tilde{\chi}_{0}, \tilde{\xi}_{0}$. For convenience we choose $C=-\tilde{\chi}_{0}$ in (88). The full metric near $r=0$ now reads

$$
\begin{aligned}
\mathrm{d} s^{2}= & -r^{2}\left(\frac{h_{0}^{2}}{\alpha_{0}^{2}}+O(r)\right)(\mathrm{d} v+O(1) \mathrm{d} \phi)^{2} \\
& \pm 2\left(\frac{1}{\sqrt{\beta_{0}}}+O(r)\right)(\mathrm{d} v+O(1) \mathrm{d} \phi) \mathrm{d} r+O(1) \mathrm{d} r^{2} \\
& +O(1)\left(\mathrm{d} \psi^{\prime}+h_{0} \cos \theta \mathrm{d} \phi^{\prime}+O\left(r^{2}\right)\right) \mathrm{d} r \\
& +2 r\left(\gamma_{0}+O(r)\right)(\mathrm{d} v+O(1) \mathrm{d} \phi)\left(\mathrm{d} \psi^{\prime}+h_{0} \cos \theta \mathrm{d} \phi^{\prime}\right) \\
& +\left(\beta_{0}+O(r)\right)\left(\mathrm{d} \psi^{\prime}+h_{0} \cos \theta \mathrm{d} \phi^{\prime}\right. \\
& \left.+O\left(r^{2}\right)\right)^{2}+\left(\alpha_{0}+O(r)\right)\left(\mathrm{d} \theta^{2}+\sin ^{2} \theta \mathrm{d} \phi^{\prime 2}\right) .
\end{aligned}
$$


The metric and its inverse are now analytic at $r=0$, hence the spacetime can be analytically extended to the region $r \leq 0$. The surface $r=0$ is an extremal Killing horizon with respect to the supersymmetric Killing field $V=\partial / \partial v$. The upper (lower) sign in $g_{v r}$ corresponds to a future (past) horizon. The gauge field near $r=0$ is given by

$$
\begin{aligned}
A= & \frac{\sqrt{3}}{2}\left[\left(\frac{h_{0}}{\alpha_{0}} r+O\left(r^{2}\right)\right) \mathrm{d} v \pm\left(\frac{\beta_{0} h_{0}-\gamma_{0}\left(h_{0} m_{0}+\frac{1}{2} k_{0} \ell_{0}\right)}{\sqrt{\beta_{0}} r}+O(1)\right) \mathrm{d} r\right. \\
& \left.+\left(\frac{h_{0} m_{0}+\frac{1}{2} k_{0} \ell_{0}}{\alpha_{0}}+O(r)\right)\left(\mathrm{d} \psi^{\prime}+h_{0} \cos \theta \mathrm{d} \phi^{\prime}\right)-\left(\tilde{\xi}_{0}-k_{0} \cos \theta+O(r)\right) \mathrm{d} \phi^{\prime}\right],
\end{aligned}
$$

so we see that the only singular terms are pure gauge. Therefore the Maxwell field $F=\mathrm{d} A$ (and hence the full solution) is analytic at $r=0$.

The near-horizon limit can be taken by transforming to coordinates $(v, r) \rightarrow(v / \epsilon, \epsilon r)$ and letting $\epsilon \rightarrow 0$, giving the near-horizon geometry

$$
\begin{aligned}
\mathrm{d} s_{\mathrm{NH}}^{2}= & -r^{2} \frac{h_{0}^{2}}{\alpha_{0}^{2}} \mathrm{~d} v^{2} \pm \frac{2}{\sqrt{\beta_{0}}} \mathrm{~d} v \mathrm{~d} r+2 r \gamma_{0} \mathrm{~d} v\left(\mathrm{~d} \psi^{\prime}+h_{0} \cos \theta \mathrm{d} \phi^{\prime}\right) \\
& +\beta_{0}\left(\mathrm{~d} \psi^{\prime}+h_{0} \cos \theta \mathrm{d} \phi^{\prime}\right)^{2}+\alpha_{0}\left(\mathrm{~d} \theta^{2}+\sin ^{2} \theta \mathrm{d} \phi^{\prime 2}\right),
\end{aligned}
$$

as well as the near-horizon Maxwell field

$$
F_{\mathrm{NH}}=\frac{\sqrt{3}}{2}\left[\frac{h_{0}}{\alpha_{0}} \mathrm{~d} r \wedge \mathrm{d} v-\left(\frac{h_{0}}{\alpha_{0}}\left(h_{0} m_{0}+\frac{1}{2} k_{0} \ell_{0}\right)+k_{0}\right) \sin \theta \mathrm{d} \theta \wedge \mathrm{d} \phi^{\prime}\right] .
$$

The second line in (97) is the metric induced on cross-sections of the horizon. For $h_{0}=0$ it is simply the standard product metric on $S^{1} \times S^{2}$. For $h_{0} \neq 0$ it is a locally homogeneous metric on $S^{3}$.

Our analysis so far in this section has been local. We will now examine the constraints imposed by asymptotic flatness. Recall in our near-horizon analysis in Sects. 3.2 and 3.3 we showed that $h_{0}=p \in \mathbb{Z}$ is the integer which fixes the horizon topology to be a lens space $L(p, q)$. The precise topology is determined by the identifications on the angles. These are already fixed by asymptotic flatness which requires $(\psi, \phi)$ to be identified as the standard Euler angles on $S^{3}$. For $p \neq 0$, it is convenient to define $\bar{\psi}=\psi^{\prime} / p$ and $\bar{\phi}=\phi^{\prime}$. From the coordinate change (88), the Killing fields are related by

$$
\left(\begin{array}{l}
\partial_{\bar{\psi}} \\
\partial_{\bar{\phi}}
\end{array}\right)=\left(\begin{array}{cc}
p & 0 \\
-\tilde{\chi}_{0} & 1
\end{array}\right)\left(\begin{array}{l}
\partial_{\psi} \\
\partial_{\phi}
\end{array}\right)
$$

and hence the matrix $A$ in (69) is determined using (30). Requiring the entries of $A$ to be integer is then equivalent to $\tilde{\chi}_{0}=p+2 n-1$ for some integer $n$. The matrix $A$ then simplifies to

$$
A=\left(\begin{array}{cc}
1-n & n \\
-p-n+1 & p+n
\end{array}\right) .
$$

By a basis change $A \rightarrow A^{\prime}=A B$ where $B \in S L(2, \mathbb{Z})$ we can bring the matrix into triangular form

$$
A^{\prime}=\left(\begin{array}{ll}
1 & 0 \\
q & p
\end{array}\right)
$$


where

$$
B=\left(\begin{array}{cc}
\alpha & -n \\
\beta & 1-n
\end{array}\right)
$$

and $(1-n) \alpha+n \beta=1$ and $q=1+p(\beta-\alpha)$. We deduce the important result

$$
q \equiv 1 \bmod p \text {. }
$$

Therefore, we have shown that the identifications that arise from asymptotic flatness, together with regularity, imply the only possible horizon topology is $L(p, 1)$. With these global identifications we find the area of cross-sections of the horizon is

$$
A=16 \pi^{2} \sqrt{-h_{0}^{2} m_{0}^{2}-3 h_{0} k_{0} \ell_{0} m_{0}+h_{0} \ell_{0}^{3}-2 k_{0}^{3} m_{0}+\frac{3}{4} k_{0}^{2} \ell_{0}^{2}},
$$

where the expression under the square root is positive (86). This completes our analysis of the horizon.

\subsection{Summary. To summarise, we have established the following results.}

Theorem 2. Consider a supersymmetric and biaxisymmetric solution to minimal supergravity containing a smooth supersymmetric horizon with compact cross-sections of topology $S^{1} \times S^{2}$ or locally $S^{3}$. In the orbit space metric the horizon is an isolated singular point on the boundary $\rho=0$, which we may take to be the origin $\rho=z=0$. Equivalently, in the Gibbons-Hawking metric, the horizon is an isolated singular point on the $z$-axis, which we may take to be the origin of $\mathbb{R}^{3}$. Furthermore, the harmonic functions can be written as

$$
H=\frac{h_{0}}{r}+H_{0}, \quad K=\frac{k_{0}}{r}+K_{0}, \quad L=\frac{\ell_{0}}{r}+L_{0}, \quad M=\frac{m_{0}}{r}+M_{0},
$$

where $r=\sqrt{\rho^{2}+z^{2}}, H_{0}, K_{0}, L_{0}, M_{0}$ are harmonic functions which are smooth at $r=0$ and $h_{0}, k_{0}, \ell_{0}, m_{0}$ are constants, where $h_{0} \neq 0$ for a locally $S^{3}$ horizon and $h_{0}=0$ for a $S^{1} \times S^{2}$ horizon. In addition, the parameters satisfy

$$
-h_{0}^{2} m_{0}^{2}-3 h_{0} k_{0} \ell_{0} m_{0}+h_{0} \ell_{0}^{3}-2 k_{0}^{3} m_{0}+\frac{3}{4} k_{0}^{2} \ell_{0}^{2}>0
$$

which in particular also implies that $h_{0} \ell_{0}+k_{0}^{2}>0$.

Theorem 3. Consider an asymptotically flat, supersymmetric and biaxisymmetric black hole solution to five-dimensional minimal supergravity.

1. Cross-sections of any connected component of the horizon must be homeomorphic to $S^{3}, S^{1} \times S^{2}$ or a lens space $L(p, 1)$.

2. The coefficient of the singular term in the harmonic function $H$ is $h_{0}= \pm 1$ for an $S^{3}$ horizon, $h_{0}=0$ for $S^{1} \times S^{2}$ and more generally $h_{0}=p \in \mathbb{Z}$ for $L(p, 1)$.

Remarks. 1. Theorem 2 is a five-dimensional analogue of Theorem 3.2 in [35] which is a crucial ingredient for the classification of supersymmetric four-dimensional black hole spacetimes.

2. The horizon is an isolated singular point of the orbit space metric also in the vacuum case. In fact this is a consequence of the $S O(2,1)$-symmetry of near-horizon geometries [45].

3. We will offer an alternative proof of part 1 of Theorem 3 by analysing the rod structure of the general solution, see Sect. 4.1. 


\section{Geometry and Topology of the Axes}

4.1. Rod structure. We now analyse the axes $\mathcal{A}$ in more detail. Recall that the axes is the part of the boundary of the orbit space $\hat{M}$ where det $\gamma_{i j}=0$, i.e. the $U(1)^{2}$-symmetry has fixed points. In Weyl coordinates $(\rho, z)$ the boundary $\partial \hat{M}$ is the $z$-axis. As shown above, a horizon corresponds to an isolated singular point on the $z$-axis. The remaining part of the $z$-axis corresponds to $\mathcal{A}$, which splits into intervals along which $\gamma_{i j}$ is of rank 1 with endpoints on which $\gamma_{i j}$ is of rank 0 . The intervals where $\gamma_{i j}$ is rank-1 correspond to the axis rods, and the endpoints where $\gamma_{i j}=0$ to the corners of the orbit space $\hat{M}$.

Lemma 4. Smoothness of the spacetime near an axis rod I implies $\hat{\omega}=O\left(\rho^{2}\right)$ and $\chi=\left.\chi\right|_{I}+O\left(\rho^{2}\right)$ where $\left.\chi\right|_{I}$ is an odd integer. The Killing field which vanishes on $I$ is

$$
v=\partial_{\phi}-\left.\chi\right|_{I} \partial_{\psi},
$$

where the normalisation has been fixed so the orbits of $v$ (away from $I$ ) are $2 \pi$-periodic.

Proof. First note that $\hat{\omega}$ and $\chi$ may be related to invariants of the metric as

$$
\hat{\omega}=\frac{1}{N}\left|\begin{array}{cc}
g_{t \psi} & g_{t \phi} \\
g_{\psi \psi} & g_{\psi \phi}
\end{array}\right|, \quad \chi=-\frac{1}{N}\left|\begin{array}{ll}
g_{t t} & g_{t \psi} \\
g_{t \phi} & g_{\psi \phi}
\end{array}\right| .
$$

These are smooth wherever $N>0$, which, by Lemma 3, includes $I$. Now, the $2 \pi$ periodic Killing field which vanishes on $I$ must be of the form

$$
v=a v_{+}+b v_{-}=(b-a) \partial_{\psi}+(a+b) \partial_{\phi},
$$

where the coefficients $(a, b)$ relative to the $2 \pi$-periodic basis $\left\{v_{+}, v_{-}\right\}$are coprime integers (not both vanishing), and where in the second equality we have used (30). Furthermore, $a+b \neq 0$, as otherwise $\partial_{\psi}=0$ which we know cannot occur on $I$, again by Lemma 3. Therefore, on $I$ we can write $\partial_{\phi}=c \partial_{\psi}$, where $c=(a-b) /(a+b)$, and hence $g_{t \phi}=c g_{t \psi}$ and $g_{\psi \phi}=c g_{\psi \psi}$. It follows that on $I$ we have $\hat{\omega}=0$ and $\chi=c$ and therefore the Killing field vanishing on $I$ is $v=(a+b)\left(\partial_{\phi}-\left.\chi\right|_{I} \partial_{\psi}\right)$, where $\left.\chi\right|_{I}=c$.

To determine the behaviour of $\hat{\omega}$ and $\chi$ near $I$ we argue as follows. First, from (33), Eq. (3) is equivalent to $\partial_{z} \chi=-\rho \partial_{\rho} H$ and $\partial_{\rho} \chi=\rho \partial_{z} H$. We know the axisymmetric harmonic function $H$ is smooth at $I$, so near $I$ we can write $H=H_{0}(z)+O\left(\rho^{2}\right)$ for some smooth function $H_{0}(z)$. Therefore, integrating it follows that $\chi=\left.\chi\right|_{I}+O\left(\rho^{2}\right)$ where $\left.\chi\right|_{I}$ is a constant. Similarly, integrating eq. (7) for $\hat{\omega}$ and using the fact that the harmonic functions $K, L, M$ are also smooth at $I$, implies $\hat{\omega}=\left.\hat{\omega}\right|_{I}+O\left(\rho^{2}\right)$ where $\left.\hat{\omega}\right|_{I}$ is a constant. Comparing to the above we deduce the constant $\left.\hat{\omega}\right|_{I}=0$.

Putting things together we find the spacetime metric for $z \in I$ and $\rho \rightarrow 0$ is

$$
\begin{aligned}
\left.\mathrm{d} s^{2}\right|_{\text {near } I}= & -f^{2}\left(\mathrm{~d} t+O\left(\rho^{2}\right) \mathrm{d} \phi_{I}\right)^{2} \\
& +\frac{H}{f}\left(\mathrm{~d} \rho^{2}+\rho^{2} \mathrm{~d} \phi_{I}^{2}+\mathrm{d} z^{2}\right)+g_{\psi \psi}\left(\mathrm{d} \psi_{I}+O\left(\rho^{2}\right) \mathrm{d} \phi_{I}\right)^{2} \\
& +2 g_{t \psi}\left(\mathrm{d} t+O\left(\rho^{2}\right) \mathrm{d} \phi_{I}\right)\left(\mathrm{d} \psi_{I}+O\left(\rho^{2}\right) \mathrm{d} \phi_{I}\right),
\end{aligned}
$$

where we have defined new coordinates $\left(\psi_{I}, \phi_{I}\right)=\left(\psi+\left.\chi\right|_{I} \phi, \phi\right)$. Now, using smoothness of the harmonic functions, $(10,16)$ implies the invariants $f, g_{\psi \psi}, g_{\psi t}, N$ must also be smooth on $I$, and near $I$ the corrections must be $O\left(\rho^{2}\right)$. Furthermore, by (17) and Lemma $3, H / f>0$ and $g_{\psi \psi}>0$ on $I$. Hence, the above is a smooth Lorentzian metric 
iff the angles are identified as $\left(\psi_{I}, \phi_{I}+2 \pi\right) \sim\left(\psi_{I}, \phi_{I}\right)$ (this can be seen by converting to cartesian coordinates in the $\left(\rho, \phi_{I}\right)$ plane). On the other hand, the identifications on the Euler angles $(\psi, \phi)$ from asymptotic flatness (30) imply the new angles are identified as $\left(\psi_{I}+4 \pi, \phi_{I}\right) \sim\left(\psi_{I}, \phi_{I}\right)$ and $\left(\psi_{I}+2 \pi\left(1+\left.\chi\right|_{I}\right), \phi_{I}+2 \pi\right) \sim\left(\psi_{I}, \phi_{I}\right)$. Compatibility of these periodicity lattices requires that $\left.\chi\right|_{I}$ is an odd integer.

Now, in terms of the new coordinates $v=(a+b) \partial_{\phi_{I}}$. We have just seen, however, that smoothness at the axis requires $\partial_{\phi_{I}}$ to have $2 \pi$-periodic orbits, so that we must have $a+b=1$. Hence in the original coordinates the Killing field which vanishes on $I$ is indeed given by (107) and $\left.\chi\right|_{I}=2 a-1$ is an odd integer.

Let us now denote the axis rods by $I_{i}=\left(z_{i}, z_{i+1}\right)$ for $i=1, \ldots, n-1$, where $z_{1}<z_{2}<\cdots<z_{n}$, and $I_{-}=\left(-\infty, z_{1}\right)$ and $I_{+}=\left(z_{n}, \infty\right)$. As we have just established, the $2 \pi$-normalised Killing fields vanishing on the respective axis rods are

$$
v_{i}=\partial_{\phi}-\chi_{i} \partial_{\psi}, \quad v_{ \pm}=\partial_{\phi}-\chi_{ \pm} \partial_{\psi} .
$$

where $\left.\chi_{i} \equiv \chi\right|_{I_{i}}$. The data $\left\{\left(I_{i}, v_{i}\right) \mid i, j=+,-, 1, \ldots, n-1\right\}$ defines the rod structure of the spacetime [20].

There are certain compatibility requirements between adjacent rods that have been derived for stationary and biaxisymmetric spacetimes [20]: If $v_{i}$ and $v_{j}$ are the $2 \pi$ normalised rod vectors of adjacent axis rods, $\operatorname{det}\left(v_{i}^{T} v_{j}^{T}\right)= \pm 1$. If two axis rods, with vectors $v_{i}$ and $v_{j}$, are separated only by a horizon, then $\operatorname{det}\left(v_{i}^{T} v_{j}^{T}\right)=p \in \mathbb{Z}$ and the topology of the horizon is $S^{1} \times S^{2}$ for $p=0, S^{3}$ for $p= \pm 1$, and in general a lens space $L(p, q)$ where $q \in \mathbb{Z}$ is only defined modulo $p$.

For asymptotically flat solutions $\chi_{ \pm}= \pm 1$ and so $v_{ \pm}$coincide with (30), thus defining a natural $2 \pi$-normalised basis. In this basis the rod vectors (111) are

$$
v_{-}=(1,0), \quad v_{i}=\left(1-a_{i}, a_{i}\right), \quad v_{+}=(0,1),
$$

where by Lemma 4 we have defined $a_{i} \equiv \frac{1}{2}\left(1+\chi_{i}\right) \in \mathbb{Z}$ for $i=1, \ldots, n-1$. The determinants of adjacent rod vectors are then given by $(i=1, \ldots, n-1)$

$$
\operatorname{det}\left(v_{-}^{T} v_{1}^{T}\right)=a_{1}, \quad \operatorname{det}\left(v_{i}^{T} v_{i+1}^{T}\right)=a_{i+1}-a_{i}, \quad \operatorname{det}\left(v_{n}^{T} v_{+}^{T}\right)=1-a_{n} .
$$

Evidently, the rod structure is somewhat restricted. In particular, this provides extra constraints on the horizon topology, thus providing an alternative proof of Theorem 3 (part 1) as follows.

Proof of Theorem 3, part 1. The proof is elementary. If $z=z_{1}$ is a horizon, the rod vectors which vanish on either side of the horizon are $v_{-}$and $v_{1}$, therefore (113) implies the horizon topology is $L\left(a_{1}, 1\right)$. Similarly, if $z=z_{n}$ is a horizon its topology is $L(1-$ $\left.a_{n}, 1\right)$. If $z=z_{i}$ is a horizon for some $i=2, \ldots, n-1$ the rod vectors which vanish on either side are $v_{i-1}$ and $v_{i}$. Thus defining

$$
P \equiv\left(v_{i-1}^{T} v_{i}^{T}\right)=\left(\begin{array}{cc}
1-a_{i-1} & 1-a_{i} \\
a_{i-1} & a_{i}
\end{array}\right)
$$

we find det $P=a_{i}-a_{i-1}=p$ where $p \in \mathbb{Z}$ and the horizon topology is $L(p, q)$. We can obtain $q$ by a performing a basis change $P \rightarrow P^{\prime}=A P$ where $A \in S L(2, \mathbb{Z})$ to put this into standard form

$$
P^{\prime}=\left(\begin{array}{ll}
1 & q \\
0 & p
\end{array}\right) \text {. }
$$


We find

$$
A=\left(\begin{array}{cc}
a & b \\
-a_{i-1} & 1-a_{i-1}
\end{array}\right)
$$

where the unit determinant condition is $1=a\left(1-a_{i-1}\right)+b a_{i-1}$. Therefore

$$
q=a\left(1-a_{i}\right)+b a_{i}=1+(b-a)\left(a_{i}-a_{i-1}\right) \equiv 1 \bmod p,
$$

where in the second equality we used the unit determinant condition. This shows the horizon topology is $L(p, 1)$ as claimed. If $p= \pm 1$ this is just $S^{3}$, and if $p=0$ this is $S^{1} \times S^{2}$.

4.2. Geometry of the axes. We now turn to the analysis of the metric on the axes. Consider an axis rod $I_{i}=\left(z_{i}, z_{i+1}\right)$ where $z=z_{i}$ is a corner of the orbit space. The induced metric on $I_{i}$ is

$$
\left.\mathrm{d} s^{2}\right|_{I_{i}}=-f^{2} \mathrm{~d} t^{2}+\frac{H}{f} \mathrm{~d} z^{2}+g_{\psi \psi} \mathrm{d} \psi_{i}^{2}+2 g_{t \psi} \mathrm{d} t \mathrm{~d} \psi_{i},
$$

where $\psi_{i}=\psi+\chi_{i} \phi$. This is a 3-dimensional timelike submanifold with a circle action generated by $\partial_{\psi}=\partial_{\psi_{i}}$ which has a fixed point at the endpoint $z=z_{i}$. At this fixed point we must have $g_{\psi \psi}=g_{t \psi}=0$. We will now analyse the conditions required by smoothness of the geometry near such a fixed point $z=z_{i}$.

It is convenient to use as a coordinate the proper distance from the fixed point

$$
s=\int_{z_{i}}^{z} \sqrt{g_{z z}} \mathrm{~d} z
$$

Now, smoothness at $z=z_{i}$ requires that $4\left(\mathrm{~d}\left|\partial_{\psi_{i}}\right|\right)^{2} \rightarrow 1$ as $z \rightarrow z_{i}^{+}$(recall that $\left.\Delta \psi_{i}=4 \pi\right)$. In terms of the proper distance this is equivalent to

$$
g_{\psi \psi}=\frac{1}{4} s^{2}\left(1+O\left(s^{2}\right)\right),
$$

where the subleading terms are fixed by smoothness at $s=0$ (and converting to cartesian coordinates in the $\left(s, \psi_{i}\right)$-plane). Smoothness of the metric and its inverse on the axis thus also requires that

$$
g_{t \psi}=O\left(s^{2}\right), \quad f^{2}=f_{i}^{2}+O\left(s^{2}\right),
$$

where $f_{i}=\left.f\right|_{z=z_{i}} \neq 0$ by Lemma 3 . This behaviour of the metric near $s=0$, together with (17), gives $f / H=\frac{1}{4} f_{i}^{2} s^{2}+O\left(s^{4}\right)$, hence using $g_{z z}=H / f$ we find

$$
z-z_{i}=\int_{0}^{s} \sqrt{\frac{f}{H}} \mathrm{~d} s=\frac{1}{4}\left|f_{i}\right| s^{2}\left(1+O\left(s^{2}\right)\right) .
$$

We deduce that

$$
H=\frac{\operatorname{sgn}\left(f_{i}\right)}{z-z_{i}}+O(1)
$$

as $z \rightarrow z_{i}^{+}$. But, by Lemma $3, H$ is a harmonic function on $\mathbb{R}^{3}$ with an isolated singularity at $(\rho, z)=\left(0, z_{i}\right)$. Thus, (123) implies that the singularity of $H$ is a pole of order one. Therefore, for $\rho \geq 0$, we must have

$$
H=\frac{h_{i}}{\sqrt{\rho^{2}+\left(z-z_{i}\right)^{2}}}+\tilde{H}_{i},
$$


where $h_{i}=\operatorname{sgn}\left(f_{i}\right)$ and $\tilde{H}_{i}$ is a harmonic function on $\mathbb{R}^{3}$ which is smooth at $(\rho, z)=$ $\left(0, z_{i}\right)$.

We will now determine the behaviour of the other harmonic functions. Since $f_{i} \neq 0$, eq. (18) implies that $\mathrm{KH}^{-1}$ is also smooth at any corner. We deduce that

$$
K=\frac{k_{i}}{\sqrt{\rho^{2}+\left(z-z_{i}\right)^{2}}}+\tilde{K}_{i},
$$

where $k_{i}$ is a constant (possibly vanishing) and $\tilde{K}_{i}$ is a harmonic function smooth at the corner $(\rho, z)=\left(0, z_{i}\right)$. Next, smoothness of $f^{-1}$ at the corner and (4) then implies $L$ may also have a pole of order one at the corner, so

$$
L=\frac{\ell_{i}}{\sqrt{\rho^{2}+\left(z-z_{i}\right)^{2}}}+\tilde{L}_{i},
$$

where $\ell_{i}=-h_{i}^{-1} k_{i}^{2}$ and $\tilde{L}_{i}$ a harmonic function smooth at the corner. Finally, the invariant $-f^{-2} V \cdot \partial_{\psi}=\omega_{\psi}$ must be smooth at any fixed point of $\partial_{\psi}$ (since $f_{i} \neq 0$ ), and thus (6) implies

$$
M=\frac{m_{i}}{\sqrt{\rho^{2}+\left(z-z_{i}\right)^{2}}}+\tilde{M}_{i},
$$

where

$$
m_{i}=-k_{i}^{3}-\frac{3}{2} h_{i}^{-1} k_{i} \ell_{i}=\frac{1}{2} k_{i}^{3}
$$

and $\tilde{M}_{i}$ is a harmonic function smooth at the corner. There are further conditions arising from the fact $\omega_{\psi}$ must also vanish at the corner which we will explore in more detail below.

Therefore, we have found that the boundary conditions arising from smoothness of the solution on the axes are sufficient to determine its functional form near any corner of the orbit space. The obtained conditions on the solution are necessary conditions for smoothness at a corner of the orbit space. In the following section we will show that in fact they are also sufficient.

4.3. Smoothness at corners of orbit space. In this section we complete the smoothness analysis at the corners of the orbit space. To do so, let us introduce $\mathbb{R}^{3}$-polar coordinates $(r, \theta, \phi)$ centred at the corner $(\rho, z)=\left(0, z_{i}\right)$, where for notational simplicity we have dropped the label $i$ in the new coordinates. Then, as just shown, we can write

$$
H=\frac{h}{r}+\tilde{H}, \quad K=\frac{k}{r}+\tilde{K}, \quad L=\frac{\ell}{r}+\tilde{L}, \quad M=\frac{m}{r}+\tilde{M},
$$

where $h= \pm 1, \ell=-h^{-1} k^{2}, m=k^{3} / 2$ and $\tilde{H}, \tilde{K}, \tilde{L}, \tilde{M}$ are axisymmetric harmonic functions that are smooth at the centre. Thus we can write

$$
\tilde{H}=\sum_{l=0}^{\infty} h_{l} r^{l} P_{l}(\cos \theta)
$$

where $P_{l}$ are the Legendre polynomials and $h_{l}$ are constants, and similarly for $\tilde{K}, \tilde{L}, \tilde{M}$, where furthermore the constants $h_{l}, k_{l}, \ell_{l}, m_{l}$ are such that $\left.\omega_{\psi}\right|_{r=0}=0$. We will now show that for asymptotically flat solutions this is sufficient for smoothness at $r=0$. 
Given (129), (130) and (33), we may solve (3) for the 1-form $\chi$, giving

$$
\chi=\left(h \cos \theta+\chi_{0}\right) \mathrm{d} \phi+\tilde{\chi},
$$

where $\chi_{0}$ is a constant and, using the fact that $P_{l}$ is a Legendre polynomial,

$$
\tilde{\chi}=r^{2} \sin ^{2} \theta \sum_{l=1}^{\infty} \frac{h_{l} r^{l-1}}{l+1} P_{l}^{\prime}(\cos \theta) \mathrm{d} \phi .
$$

Now, define new coordinates $\left(R, \psi^{\prime}, \phi^{\prime}\right)$ by

$$
r=\frac{1}{4} R^{2}, \quad \psi^{\prime}=\psi+\chi_{0} \phi, \quad \phi^{\prime}=h \phi,
$$

so that the $\mathrm{GH}$ base is

$$
\mathrm{d} s_{G H}^{2}=F\left(\mathrm{~d} R^{2}+\frac{1}{4} R^{2}\left[\left(\mathrm{~d} \theta^{2}+\sin ^{2} \theta \mathrm{d} \phi^{2}\right)+\frac{1}{F^{2}}\left(\mathrm{~d} \psi^{\prime}+\cos \theta \mathrm{d} \phi^{\prime}+\tilde{\chi}\right)^{2}\right]\right)
$$

where we have defined $F \equiv r H=h+r \tilde{H}$. In terms of the new coordinates

$$
F= \pm 1+O\left(R^{2}\right), \quad \tilde{\chi}=O\left(R^{4}\right) \mathrm{d} \phi^{\prime},
$$

so we see that as $R \rightarrow 0$, the GH base approaches the origin $\pm \mathbb{R}^{4}$, if the angles $\left(\psi^{\prime}, \phi^{\prime}\right)$ are identified as Euler angles on $S^{3}$. Since the original angles $(\psi, \phi)$ are required to be Euler angles on $S^{3}$ by asymptotic flatness, it is easy to see that $\left(\psi^{\prime}, \phi^{\prime}\right)$ are also Euler angles on $S^{3}$ if and only if $\chi_{0}=h-2 n-1$ for some $n \in \mathbb{Z}$. In fact, this condition follows from Lemma 4: On the axis $\theta=0, \pi$ it is clear that $\tilde{\chi}=0$ and thus on any axis rod $I$ we have $\left.\chi\right|_{I}=\chi_{0} \pm h$. But by Lemma 4, we know that $\left.\chi\right|_{I}$ is an odd integer and hence $\chi_{0}$ is an even integer as required.

In order to verify the GH base at the centre is actually smooth requires us to control the higher order terms more carefully. To this end introduce coordinates ${ }^{8}$

$$
\phi^{ \pm}=\frac{1}{2}\left(\psi^{\prime} \pm \phi^{\prime}\right), \quad X_{+}=R \cos \left(\frac{1}{2} \theta\right), \quad X_{-}=R \sin \left(\frac{1}{2} \theta\right)
$$

so

$$
\mathrm{d} s^{2}\left(\mathbb{R}^{4}\right)=\mathrm{d} X_{+}^{2}+X_{+}^{2}\left(\mathrm{~d} \phi^{+}\right)^{2}+\mathrm{d} X_{-}^{2}+X_{-}^{2}\left(\mathrm{~d} \phi^{-}\right)^{2}
$$

and $\phi^{ \pm}$are independently $2 \pi$ periodic. In these coordinates any smooth biaxisymmetric function on $\mathbb{R}^{4}$ is a smooth function of $\left(X_{+}^{2}, X_{-}^{2}\right)$. Noting that

$$
r=\frac{1}{4}\left(X_{+}^{2}+X_{-}^{2}\right), \quad r \cos \theta=\frac{1}{4}\left(X_{+}^{2}-X_{-}^{2}\right)
$$

and using the fact that $P_{l}$ are polynomials of order $l$, it is easy to see that $\tilde{H}$ and hence $F$ are analytic functions of $\left(X_{+}^{2}, X_{-}^{2}\right)$. Similarly we find that

$$
\tilde{\chi}=\frac{1}{4} X_{+}^{2} X_{-}^{2}\left(h_{1}+\cdots\right) h \mathrm{~d} \phi^{\prime}=\frac{1}{4} X_{+}^{2} X_{-}^{2}\left(h_{1}+\cdots\right) h\left(\mathrm{~d} \phi^{+}-\mathrm{d} \phi^{-}\right)
$$

\footnotetext{
8 The coordinates $\phi^{ \pm}$in this section are different to those in (30). We will only use these in this section, so there should be no confusion.
} 
where the higher order terms are analytic in $\left(X_{+}^{2}, X_{-}^{2}\right)$, so the 1-form $\tilde{\chi}$ is analytic at the origin of $\mathbb{R}^{4}$. Putting everything together, we can write the GH base as

$$
\begin{aligned}
\mathrm{d} s_{G H}^{2}= & F \mathrm{~d} s^{2}\left(\mathbb{R}^{4}\right)-\frac{\tilde{H}(h+F)}{4 F}\left(X_{+}^{2} \mathrm{~d} \phi^{+}+X_{-}^{2} \mathrm{~d} \phi^{-}\right)^{2} \\
& +\frac{1}{F}\left(X_{+}^{2} \mathrm{~d} \phi^{+}+X_{-}^{2} \mathrm{~d} \phi^{-}\right) \tilde{\chi}+\frac{F\left(X_{+}^{2}+X_{-}^{2}\right)}{4} \tilde{\chi}^{2}
\end{aligned}
$$

which is now manifestly analytic at the origin of $\mathbb{R}^{4}$. Therefore, the $\mathrm{GH}$ base is indeed smooth, in fact analytic, at any centre corresponding to a corner of the orbit space.

We now turn to the other components of the spacetime metric, namely the function $f$ and 1-form $\omega$. Expanding the regular parts $\tilde{K}, \tilde{L}, \tilde{M}$ of the harmonic functions $K, L, M$ as above for $\tilde{H}$ it is easy to see that $f$ is an analytic function of $\left(X_{+}^{2}, X_{-}^{2}\right)$. Recall by Lemma 3 we must have $f \neq 0$ at any centre corresponding to a corner of the orbit space.

It remains to be checked that also the 1 -form $\omega$ is smooth at the centre. In the above coordinates we can write

$$
\omega=\omega_{\psi}\left(\mathrm{d} \psi^{\prime}+\cos \theta \mathrm{d} \phi^{\prime}\right)+\omega_{\psi} \tilde{\chi}+\hat{\omega}=\frac{2 \omega_{\psi}}{R^{2}}\left(X_{+}^{2} \mathrm{~d} \phi^{+}+X_{-}^{2} \mathrm{~d} \phi^{-}\right)+\omega_{\psi} \tilde{\chi}+\hat{\omega} .
$$

Using (6) and expanding

$$
\frac{1}{h+r \tilde{H}}=h-r \tilde{H}+r^{2} G_{1}
$$

where $G_{1}=\tilde{H}^{2} /(h+r \tilde{H})$ is analytic in $\left(X_{+}^{2}, X_{-}^{2}\right)$, as well as making use of the identities $h^{2}=1, \ell=-h k^{2}, m=k^{3} / 2$, one finds

$$
\omega_{\psi}=\sum_{l=0}^{\infty}\left(m_{l}-h m h_{l}+\frac{3}{2}\left(h k \ell_{l}-h \ell k_{l}\right)\right) r^{l} P_{l}(\cos \theta)+r \tilde{G}_{1},
$$

where $\tilde{G}_{1}$ is some analytic function in $\left(X_{+}^{2}, X_{-}^{2}\right)$. Thus $\omega_{\psi}$, and hence also $\omega_{\psi} \tilde{\chi}$ are analytic in $\left(X_{+}^{2}, X_{-}^{2}\right)$ and for smoothness of (141) we therefore only need to check that

$$
\left(\frac{2 \omega_{\psi} X_{+}^{2}}{R^{2}}+h \hat{\omega}_{\phi}\right) \mathrm{d} \phi^{+}+\left(\frac{2 \omega_{\psi} X_{-}^{2}}{R^{2}}-h \hat{\omega}_{\phi}\right) \mathrm{d} \phi^{-}
$$

is smooth at the origin, or equivalently that

$$
\frac{2 \omega_{\psi} X_{ \pm}^{2}}{R^{2}} \pm h \hat{\omega}_{\phi}=X_{ \pm}^{2} G_{ \pm}
$$

for some smooth functions $G_{ \pm}$of $\left(X_{+}^{2}, X_{-}^{2}\right)$.

In fact one can solve Eq. (7) for the 1-form $\hat{\omega}$, of the form (33), as

$$
\hat{\omega}_{\phi}=\hat{\omega}_{0}+r \sin ^{2} \theta \sum_{l=1}^{\infty} \frac{\left(h m_{l}-h_{l} m\right)+\frac{3}{2}\left(k \ell_{l}-k_{l} \ell\right)}{l} r^{l-1} P_{l}^{\prime}(\cos \theta)+h r^{2} \sin ^{2} \theta G_{2},
$$


where we have used that $\left.\omega_{\psi}\right|_{r=0}=0$ and defined

$$
G_{2}=h \sum_{j=0}^{\infty} \sum_{l=1}^{\infty} \frac{h_{j} m_{l}-h_{l} m_{j}+\frac{3}{2}\left(k_{j} \ell_{l}-k_{l} \ell_{j}\right)}{l+j+1} r^{l+j-1} P_{l}^{\prime}(\cos \theta) P_{j}(\cos \theta)
$$

which is an analytic function of $\left(X_{+}^{2}, X_{-}^{2}\right)$. From Lemma 4 , we must have $\hat{\omega}_{0}=0$. Then

$$
\begin{aligned}
& \frac{2 \omega_{\psi} X_{ \pm}^{2}}{R^{2}} \pm h \hat{\omega}_{\phi}=\frac{X_{ \pm}^{2}}{2} \tilde{G}_{1} \pm \frac{X_{+}^{2} X_{-}^{2}}{4} G_{2} \\
& +h \sum_{l=1}^{\infty}\left(h m_{l}-m h_{l}+\frac{3}{2}\left(k \ell_{l}-\ell k_{l}\right)\right) r^{l}\left((1 \pm \cos \theta) P_{l}(\cos \theta) \pm \frac{\sin ^{2} \theta}{l} P_{l}^{\prime}(\cos \theta)\right)
\end{aligned}
$$

and it is obvious that the first two terms are of the required form. Using basic properties of Legendre polynomials we can rewrite

$$
(1 \pm \cos \theta) P_{l}(\cos \theta) \pm \frac{\sin ^{2} \theta}{l} P_{l}^{\prime}(\cos \theta)=P_{l}(\cos \theta) \pm P_{l-1}(\cos \theta) .
$$

Furthermore, from the recursion formula for Legendre polynomials, it follows that 9

$$
r^{l}\left[\left(P_{l}(\cos \theta) \pm P_{l-1}(\cos \theta)\right]=r(1 \pm \cos \theta) \tilde{G}_{ \pm}=\frac{X_{ \pm}^{2}}{2} \tilde{G}_{ \pm}\right.
$$

for some analytic $\tilde{G}_{ \pm}$, so indeed (145) is satisfied. This establishes that the 1 -form $\omega$ is smooth, in fact analytic, at any centre corresponding to a corner of the orbit space.

Putting things together, we have shown that the spacetime metric is analytic at any point corresponding to a corner of the orbit space. Furthermore, near such points the spacetime is diffeomorphic to $\mathbb{R}^{1,4}$.

The gauge field in the new coordinates takes the form

$$
A=\frac{\sqrt{3}}{2}\left(f \mathrm{~d} t+A_{+} \mathrm{d} \phi^{+}+A_{-} \mathrm{d} \phi^{-}\right)
$$

where

$$
A_{ \pm}=f\left(\frac{2 \omega_{\psi} X_{ \pm}^{2}}{R^{2}} \pm h \hat{\omega}_{\phi}\right) \pm h\left(f \omega_{\psi}-\frac{K}{H}\right) \tilde{\chi}_{\phi}-\frac{2 X_{ \pm}^{2}}{R^{2}} \frac{K}{H} \mp h \xi_{\phi} .
$$

Clearly $A_{t}$ is analytic at $R=0$. We have already shown (145), so the first term in (152) is analytic and proportional to $X_{ \pm}^{2}$. As $f, \omega_{\psi}, K / H$ are analytic at the centre and $\tilde{\chi}$ is of the form (139), the same is true for the second term. Lastly, integrating (9), for $\xi$ of the form (33), gives

$$
\xi=\left(\xi_{0}-k \cos \theta-\frac{X_{+}^{2} X_{-}^{2}}{4} \sum_{l=1}^{\infty} \frac{k_{l} r^{l-1}}{l+1} P_{l}^{\prime}(\cos \theta)\right) \mathrm{d} \phi
$$

\footnotetext{
9 This follows easily by induction from writing the recursion formula in the form $(l+1)\left(P_{l+1} \pm P_{l}\right)=$ $\mp l\left(P_{l} \pm P_{l-1}\right) \pm(1 \pm \cos \theta)(2 l+1) P_{l}$ and noting that $P_{1} \pm P_{0}=\cos \theta \pm 1$.
} 
and hence, using (142),

$$
-\frac{2 X_{ \pm}^{2}}{R^{2}} \frac{K}{H} \mp h \xi_{\phi}=-h k \mp h \xi_{0}+X_{ \pm}^{2}(\ldots)+X_{+}^{2} X_{-}^{2}(\ldots),
$$

where ... are analytic functions of $\left(X_{+}^{2}, X_{-}^{2}\right)$. Thus $A$ is gauge-equivalent to an analytic 1 -form. Therefore the Maxwell field (hence the full solution) is analytic at the centre.

Finally, we emphasise that the above analysis shows that the solution is smooth and stably causal at and near any centre corresponding to a corner of the orbit space. Indeed we have,

$$
K^{2}+H L=\frac{1}{|f| r}+O(1), \quad g^{t t}=-\frac{1}{f^{2}}+O(r),
$$

where recall that $f \neq 0$ at the centre, thus confirming the solution is smooth (23) and causal (25) near the centre.

\subsection{Summary. To summarise, we have shown the following.}

Theorem 4. Let $(M, g, F)$ be an asymptotically flat, supersymmetric and biaxisymmetric solution to minimal supergravity with a globally hyperbolic domain of outer communication $\langle\langle M\rangle\rangle$. Let $(\rho, z)=\left(0, z_{i}\right)$ be a point corresponding to a corner of the orbit space. Then the solution is smooth (in fact analytic) at the corner if and only if $\left.f_{i} \equiv f\right|_{(\rho, z)=\left(0, z_{i}\right)} \neq 0,\left.\omega_{\psi}\right|_{(\rho, z)=\left(0, z_{i}\right)}=0$ and the harmonic functions $H, K, L, M$ are given by

$$
H=\frac{h_{i}}{r_{i}}+\tilde{H}_{i}, \quad K=\frac{k_{i}}{r_{i}}+\tilde{K}_{i}, \quad L=-\frac{h_{i}^{-1} k_{i}^{2}}{r_{i}}+\tilde{L}_{i}, \quad M=\frac{\frac{1}{2} k_{i}^{3}}{r_{i}}+\tilde{M}_{i},
$$

where $r_{i}=\sqrt{\rho^{2}+\left(z-z_{i}\right)^{2}}, h_{i}=\operatorname{sgn}\left(f_{i}\right), k_{i}$ are constants and $\tilde{H}_{i}, \tilde{K}_{i}, \tilde{L}_{i}, \tilde{M}_{i}$ are harmonic functions on $\mathbb{R}^{3}$ which are smooth at $(\rho, z)=\left(0, z_{i}\right)$. Furthemore, the spacetime near such a corner is diffeomorphic to $\mathbb{R}^{1,4}$.

\section{Moduli Space of Soliton and Black Hole Solutions}

5.1. Classification theorem. We will now combine the constraints obtained from the existence of a smooth horizon in Sect. 3 and smooth axes in Sect. 4 and give our main classification theorem.

Theorem 5. Consider an asymptotically flat, supersymmetric and biaxisymmetric solution to minimal supergravity with a smooth globally hyperbolic domain of outer communication and a smooth event horizon with compact cross-sections (if there is a black hole). Suppose the orbit space $\hat{M}$ has $k$ corners and the horizon has $l$ connected components $(l=0$ corresponds to no black hole $)$, and let $n=k+l$. Then, the harmonic functions are

$$
H=\sum_{i=1}^{n} \frac{h_{i}}{r_{i}}, \quad K=\sum_{i=1}^{n} \frac{k_{i}}{r_{i}}, \quad L=1+\sum_{i=1}^{n} \frac{\ell_{i}}{r_{i}}, \quad M=m+\sum_{i=1}^{n} \frac{m_{i}}{r_{i}},
$$


where $r_{i}=\sqrt{\rho^{2}+\left(z-z_{i}\right)^{2}},(\rho, z)=\left(0, z_{i}\right)$ are corners of the orbit space or horizons, and

$$
\sum_{i=1}^{n} h_{i}=1, \quad m=-\frac{3}{2} \sum_{i=1}^{n} k_{i}
$$

The corresponding 1-forms can be written as

$$
\begin{aligned}
\chi= & \sum_{i=1}^{n} \frac{h_{i}\left(z-z_{i}\right)}{r_{i}} \mathrm{~d} \phi, \quad \xi=-\sum_{i=1}^{n} \frac{k_{i}\left(z-z_{i}\right)}{r_{i}} \mathrm{~d} \phi, \\
\hat{\omega}= & {\left[-\sum_{i=1}^{n} \frac{\left(m h_{i}+\frac{3}{2} k_{i}\right)\left(z-z_{i}\right)}{r_{i}}\right.} \\
& \left.-\sum_{i=1}^{n} \sum_{j \neq i}\left(\frac{h_{i} m_{j}+\frac{3}{2} k_{i} \ell_{j}}{z_{i}-z_{j}}\right)\left(\frac{\rho^{2}+\left(z-z_{i}\right)\left(z-z_{j}\right)}{r_{i} r_{j}}-1\right)\right] \mathrm{d} \phi,
\end{aligned}
$$

and the parameters have to satisfy for each $i=1, \ldots, n$,

$$
h_{i} m+\frac{3}{2} k_{i}+\sum_{\substack{j=1 \\ j \neq i}}^{n} \frac{h_{i} m_{j}-m_{i} h_{j}-\frac{3}{2}\left(\ell_{i} k_{j}-k_{i} \ell_{j}\right)}{\left|z_{i}-z_{j}\right|}=0 .
$$

Furthermore, if $\left(0, z_{i}\right)$ is a corner, $h_{i}= \pm 1$ and the parameters must satisfy

$$
\begin{aligned}
& \ell_{i}=-h_{i}^{-1} k_{i}^{2}, \quad m_{i}=\frac{1}{2} k_{i}^{3}, \\
& h_{i}+\sum_{\substack{j=1 \\
j \neq i}}^{n} \frac{2 k_{i} k_{j}-h_{i}\left(h_{j} k_{i}^{2}-\ell_{j}\right)}{\left|z_{i}-z_{j}\right|}>0 .
\end{aligned}
$$

On the other hand, if $\left(0, z_{i}\right)$ is a horizon the parameters must satisfy $h_{i} \in \mathbb{Z}$,

$$
-h_{i}^{2} m_{i}^{2}-3 h_{i} k_{i} \ell_{i} m_{i}+h_{i} \ell_{i}^{3}-2 k_{i}^{3} m_{i}+\frac{3}{4} k_{i}^{2} \ell_{i}^{2}>0,
$$

(which also implies $h_{i} \ell_{i}+k_{i}^{2}>0$ ) and cross-sections of the horizon are of topology $S^{3}$ if $h_{i}= \pm 1, S^{2} \times S^{1}$ if $h_{i}=0$ and the lens space $L\left(h_{i}, 1\right)$ otherwise.

Proof. We have shown that a horizon corresponds to at most a simple pole of the harmonic functions $H, K, L, M$, see Theorem 2. Similarly, a corner corresponds to a simple pole of $H$ and at most a simple pole of $K, L, M$, see Theorem 4. Hence, with the stated assumptions we can write

$$
H=\tilde{H}+\sum_{i=1}^{n} \frac{h_{i}}{r_{i}}, \quad K=\tilde{K}+\sum_{i=1}^{n} \frac{k_{i}}{r_{i}}, \quad L=\tilde{L}+\sum_{i=1}^{n} \frac{\ell_{i}}{r_{i}}, \quad M=\tilde{M}+\sum_{i=1}^{n} \frac{m_{i}}{r_{i}},
$$

where $\tilde{H}, \tilde{K}, \tilde{L}, \tilde{M}$ are harmonic functions smooth at $(\rho, z)=\left(0, z_{i}\right)$ for all $i=$ $1, \ldots, n$. By Lemma 3 , the only singularities of $H, K, L, M$ in the DOC are at points corresponding to the corners of the orbit space, or at the horizon. Therefore, $\tilde{H}, \tilde{K}, \tilde{L}, \tilde{M}$ must be smooth on all of $\mathbb{R}^{3}$. Asymptotic flatness (31) implies these harmonic functions 
are bounded. Therefore, $\tilde{H}, \tilde{K}, \tilde{L}, \tilde{M}$ are smooth and bounded harmonic functions on $\mathbb{R}^{3}$. Therefore, they must be constants which coincide with their asymptotic values, so $\tilde{H}=0, \tilde{L}=1, \tilde{K}=0$ and $\tilde{M}=m$. The asymptotic flatness conditions (31) and (32) then reduce to (158). This establishes the form of the harmonic functions.

Given the harmonic functions the 1 -forms are easily integrated using $(3,7,9)$. The integration constants in $\chi$ and $\hat{\omega}$ have been fixed so that $\chi=\cos \theta+O\left(r^{-1}\right)$ and $\hat{\omega}=O\left(r^{-1}\right)$ as $r \rightarrow \infty$, as required by asymptotic flatness.

The constraints on the parameters at each corner (161) are given in Theorem 4 . The additional constraint (162) is equivalent to the condition $h_{i} f_{i}>0$, which also follows from Theorem 4. The constraints on the parameters at a horizon (163) are given in Theorems 2 and 3.

The constraints (160) are equivalent to $\hat{\omega}=0$ on each of the axis rods $I_{i}=\left(z_{i}, z_{i+1}\right)$, which is required by smoothness at the axes, see Lemma 4 . This can be seen as follows. From (159) it is obvious that $\hat{\omega}$ is constant on any axis rod $I_{i}$. It can be shown that the difference between $\hat{\omega}$ evaluated on two adjacent rods separated by the centre $\left(0, z_{i}\right)$ is given by -2 times the lefthand side of (160). Furthermore, by asymptotic flatness $\hat{\omega}$ vanishes on the rods $I_{+}=\left(z_{n}, \infty\right)$ and $I_{-}=\left(-\infty, z_{1}\right)$. We deduce that $\left.\hat{\omega}\right|_{I_{i}}=0$ for all $i=1, \ldots, n-1$ precisely if (160) is satisfied, as claimed. It is worth noting that for any corner $\left(0, z_{i}\right)$ the condition $(160)$ is in fact equivalent to $\left.\omega_{\psi}\right|_{r_{i}=0}=0$, as is also required by Theorem 4 .

Finally, we note that the other condition required for smooth axes, given in Lemma 4 , is that $\chi$ evaluated on each axis rod has to be an odd integer. Evaluating (159) on each axis rod, we find this is automatically satisfied since $h_{i}$ are integers for all $i=1, \ldots, n$ (see equation (173)).

Remarks. 1. This shows that supersymmetric black holes and solitons, must be multicentred solutions with a Gibbons-Hawking base. This is a five-dimensional analogue of Corollary 4.2 in [35].

2. To confirm that the solution is smooth and stably causal everywhere in the DOC one must check the condition (23) in Lemma 1 and (25). Our analysis shows that these are indeed satisfied near infinity, near the horizon and near any point corresponding to a corner of the orbit space. We have been unable to check that the conditions listed in Theorem 5 are sufficient to ensure smoothness and causality are obeyed everywhere else in the DOC. However, based on the known examples (discussed below) we believe that no further conditions on the parameters arise. Nevertheless, it is possible that (23) and (25) may impose additional constraints on the parameters of the solution.

We can see from Theorem 5 that a general solution with $n=k+l$ centres will be determined by the (discrete) $n$-dimensional vector $h=\left(h_{1}, \ldots, h_{n}\right)$, as well as $(4 n-1)$ real parameters,

$$
\left\{\left\{z_{i+1}-z_{i}\right\}_{i=1, \ldots, n-1},\left\{k_{i}, \ell_{i}, m_{i}\right\}_{i=1, \ldots, n}\right\}
$$

subject to $3 k+l$ constraint equations (160)-(161), of which (161) can be solved algebraically. Furthermore, there is a remaining one-parameter gauge freedom (21)-(22) in the harmonic functions under which

$$
k_{i} \rightarrow k_{i}+c h_{i}, \quad \ell_{i} \rightarrow \ell_{i}-2 c k_{i}-2 c^{2} h_{i}, \quad m_{i} \rightarrow m_{i}-\frac{3}{2} c \ell_{i}+\frac{3}{2} c^{2} k_{i}+\frac{1}{2} c^{3} h_{i}
$$


Summing up, we find that the moduli space of $(k+l)$-centred solutions, $\mathcal{M}^{k, l}$, is given by the subset of the $(2 k+4 l-1)$-dimensional parameter space

$$
\left\{\left\{z_{i+1}-z_{i}\right\}_{i=1, \ldots, n-1},\left\{k_{i}\right\}_{i=1, \ldots, n},\left\{\ell_{j}, m_{j}\right\}_{\text {if } z_{j}} \text { is a horizon }\right\},
$$

defined by the set of $k+l$ polynomial equations (160) subject to the inequalities (162) and equivalence relations (166). By a general count of degrees of freedom,

$$
\operatorname{dim} \mathcal{M}^{k, l}=k+3 l-2-\tilde{\Delta}+\Delta(k, l),
$$

where $\tilde{\Delta}$ has been introduced to correct for any potential restrictions on the parameters coming from $(23,25)$ (see Remark 2 above), and the second correction term $\Delta(k, l)$ to accomodate for a potential redundancy in equations (160). One can easily see that there is at least one such redundancy as summing (160) over all $i$ gives

$$
m \sum_{i=1}^{n} h_{i}+\frac{3}{2} \sum_{i=1}^{n} k_{i}+\sum_{i=1}^{n} \sum_{\substack{j=1 \\ j \neq i}}^{n} \frac{h_{i} m_{j}-m_{i} h_{j}-\frac{3}{2}\left(\ell_{i} k_{j}-k_{i} \ell_{j}\right)}{\left|z_{i}-z_{j}\right|}=0
$$

where we have made use of (158) and (161), and the double sum vanishes for reasons of symmetry. We thus know that

$$
1 \leq \Delta(k, l) \leq k+l .
$$

In fact on the basis of known examples, we will conjecture that $\tilde{\Delta}=0, \Delta(k, l)=1$, so

$$
\operatorname{dim} \mathcal{M}^{k, l}=k+3 l-1
$$

Indeed, this agrees with the known solutions which are discussed below.

When counting the number of solutions it is important to realise there is a redundancy in our parameterisation corresponding to a discrete global isometry,

$$
z \rightarrow-z, \quad z_{i} \rightarrow-z_{n-i+1}, \quad \phi \rightarrow-\phi, \quad i \rightarrow n-i+1 .
$$

Now, each separate choice of $h$ will define a component of the moduli space. The number of connected components of $\mathcal{M}^{k, l}$ is thus given by the number of possible choices of $h$, taking into account the remaining reflection symmetry (172) of the axis. As we will show next, the choice of $h$ is precisely equivalent to the rod structure of the solution, so the number of components of the moduli space is also the number of inequivalent rod structures.

As we have seen earlier, the centres $z=z_{i}$ split the $z$-axis into $n+1$ intervals, $I_{ \pm}, I_{i}$, on each of which the respective Killing field (111) vanishes. Having the full solution at hand, we can now explicitly evaluate $\chi$ on each of these intervals as

$$
\chi_{ \pm}= \pm 1,\left.\quad \chi_{i} \equiv \chi\right|_{I_{i}}=\sum_{j=1}^{i} h_{j}-\sum_{j=i+1}^{n} h_{j}=2 \sum_{j=1}^{i} h_{j}-1
$$

where the final equality follows from the asymptotic condition (158). Therefore, in the basis defined by (30), one finds the rod vectors are given by (112) with

$$
a_{i}=\sum_{j=1}^{i} h_{j}
$$




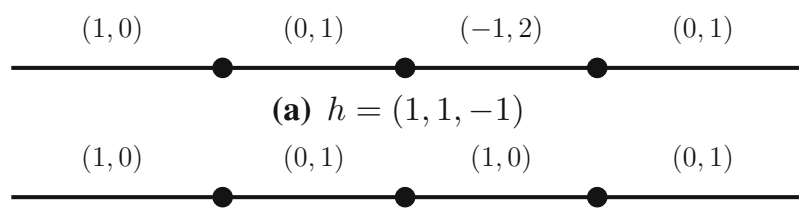

(b) $h=(1,-1,1)$

Fig. 1. Inequivalent rod structures for 3-centred solitons

The determinants of adjacent rod vectors (113) are then precisely given by the value of $h_{i}$ at the respective centre,

$$
\operatorname{det}\left(v_{-}^{T} v_{1}^{T}\right)=h_{1}, \quad \operatorname{det}\left(v_{i}^{T} v_{i+1}^{T}\right)=h_{i+1}, \quad \operatorname{det}\left(v_{n}^{T} v_{+}^{T}\right)=h_{n} .
$$

Therefore, our horizon and axes analysis, which showed that $h_{i}=p$ for a centre corresponding to an $L(p, q)$ horizon, and $h_{i}= \pm 1$ for a centre corresponding to a corner of the orbit space, precisely agree with the compatibility conditions for adjacent rod vectors previously derived for stationary and biaxisymmetric spacetimes [20]. Therefore, these compatibility conditions impose no extra constraints.

The topology of the domain of outer communication is nontrivial and determined by the rod structure. The internal axis rods $I_{i}(i=1, \ldots, n-1)$, or indeed any simple curve in the $\mathbb{R}^{3} \mathrm{GH}$ base between the endpoints of $I_{i}$, together with the $U(1) \psi$-fibre over the $\mathrm{GH}$ base, correspond to noncontractible 2-cycles $C_{i}$. If the endpoints of $I_{i}$ are both corners of the orbit space the $\psi$-fibre collapses smoothly at the endpoints, so $C_{i}$ is a surface of $S^{2}$ topology. If one endpoint of $I_{i}$ is a corner and one a horizon, then $C_{i}$ is a surface of 2-disc topology, with the boundary of the disc attached to the horizon. Finally, if both endpoints are horizons then $C_{i}$ is a 2-tube with each of its boundaries attached to one horizon.

5.2. Soliton solutions. Let us first consider the moduli space of $n$-centred soliton solutions, $\mathcal{M}^{n, 0}$. Since every centre corresponds to a corner of the orbit space we must have $h_{i}= \pm 1$ for all $i=1, \ldots n$. On the other hand, asymptotic flatness requires $\sum_{i=1}^{n} h_{i}=1$. It follows that soliton solutions will necessarily have an odd number of centres, $n=2 m+1$, where $m$ is the number of $h_{i}=-1$. We can now easily determine the number of distinct rod structures this allows for. There are $\left(\begin{array}{l}n \\ m\end{array}\right)$ possible ways of choosing $h \equiv\left(h_{1}, \ldots, h_{n}\right)$. Some of these, however, will be related by the discrete reflection symmetry (172) (which implies $h_{i} \rightarrow h_{n-i+1}$ ) and thus correspond to isometric solutions. Correcting for this overcounting, one finds the number of connected components of the moduli space to be given by

$$
N\left(\mathcal{M}^{n, 0}\right)=\frac{1}{2}\left[\left(\begin{array}{l}
n \\
m
\end{array}\right)+\left(\begin{array}{c}
m \\
{[m / 2]}
\end{array}\right)\right],
$$

where the latter term arises as a correction for solutions which are themselves symmetric under reflection (and thus had not falsely been overcounted before).

For $n=1$, the only possible solution without a black hole is Minkowski space. The allowed inequivalent rod structures for $n=3$ are defined by $h=(1,1,-1)$ and $h=(1,-1,1)$ and are depicted in Fig. 1 . 
In particular, we see that there are two inequivalent soliton solutions in this case. The above counting formula shows that the number of inequivalent soliton solutions increases with $n$.

The $n$-centred soliton solutions correspond to asymptotically flat, globally hyperbolic regular spacetimes containing $n-1$ noncontracible 2-cycles, or 'bubbles'. Such bubbling spacetimes were first constructed in [39] and some their global properties elucidated in [4].

5.3. Single black hole solutions. We now consider the moduli space for $n$-centred solutions with a single black hole, $\mathcal{M}^{n-1,1}$. Thus, for one centre, say $z_{j}$, the determinant of the matrix of adjacent rod vectors $h_{j}=p \in \mathbb{Z}$ while the other centres correspond to corners, so $h_{i}= \pm 1$ for all $i \neq j$. As we have seen, this means that the centre $z=z_{j}$ corresponds to a horizon of topology $L(p, 1)$. Denote the number of corners with $h_{i}= \pm 1$ by $n_{ \pm}$so $n_{+}+n_{-}+1=n$. Asymptotic flatness (158) also implies that $n_{+}-n_{-}+p=1$. It follows that

$$
p=n-2 n_{+}
$$

where $0 \leq n_{+} \leq n-1$. Hence $p$ is even for an even number of centres and odd otherwise and the possible values of $p$ are $-n+2,-n+4, \ldots, n-2, n$.

For a given $p$, there are $n\left(\begin{array}{c}n-1 \\ n_{+}\end{array}\right)$ways of choosing $h$. However, some of these configurations will be related by the reflection symmetry (172) and hence they are double counted. To determine this number, we first must identify the number of configurations which are symmetric under the reflection. Symmetric rod structures can only occur for odd $n$ and even $n_{+}$, with the middle centre corresponding to the horizon, in which case there are $\left(\begin{array}{c}(n-1) / 2 \\ n_{+} / 2\end{array}\right)$ such symmetric configurations. Putting all this together, we find that the number of components of the moduli space of single black hole solutions with $L(p, 1)$ topology, $\mathcal{M}_{p}^{n-1,1}$, is

$$
N\left(\mathcal{M}_{p}^{n-1,1}\right)= \begin{cases}\frac{n}{2}\left(\begin{array}{c}
n-1 \\
n_{+}
\end{array}\right)+\frac{1}{2}\left(\begin{array}{c}
(n-1) / 2 \\
n_{+} / 2
\end{array}\right) & \text { if } n \text { odd and } n_{+} \text {even } \\
\frac{n}{2}\left(\begin{array}{c}
n-1 \\
n_{+}
\end{array}\right) & \text {otherwise }\end{cases}
$$

Summing over the possible $p$ we find that the total number is

$$
N\left(\mathcal{M}^{n-1,1}\right)=\sum_{n_{+}=0}^{n-1} N\left(\mathcal{M}_{p}^{n-1,1}\right)= \begin{cases}n 2^{n-2} & \text { if } n \text { is even } \\ n 2^{n-2}+2^{\frac{n-3}{2}} & \text { if } n \text { is odd }\end{cases}
$$

Let us consider a few examples.

The simplest possibility is $n=1$, which implies $n_{+}=0$ and $p=1$ and hence the horizon topology is $S^{3}$. This of course corresponds to the BMPV black hole [34].

Now let us consider the $n=2$ case. From (179) we find that there are 2 classes of two-centred single black hole solutions, whose rod structures are shown in Fig. 2. The first of these, Fig. 2a is the recently constructed $L(2,1)$ black lens [8]. Figure $2 \mathrm{~b}$ corresponds to the known supersymmetric black ring solution [37].

Next, for $n=3$ we see there are seven distinct rod structures. These are depicted in Figs. 3 and 4. There are two inequivalent black holes with a horizon of topology $L(3,1)$, of which only Fig. 3a corresponds to the solution constructed in [9]. There are five inequivalent $S^{3}$ black holes, of which only Fig. 3b corresponds to the known $S^{3}$ black hole with bubble [6]. The other solutions had not previously been constructed. 


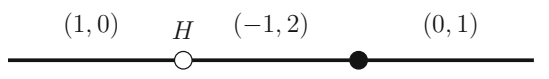

(a) $p=2, h=(2,-1)$

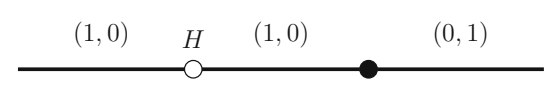

(b) $p=0, h=(0,1)$.

Fig. 2. Rod structures for 2-centred single black hole solutions

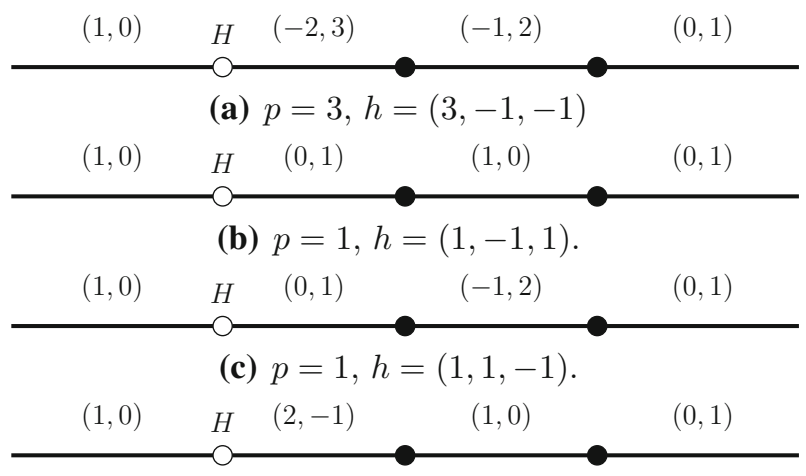

(d) $p=-1, h=(-1,1,1)$.

Fig. 3. Rod structures for 3-centred single black holes with the horizon at the first centre

$\begin{array}{llll}\text { (a) } p=3, h=(-1,3,-1) & (0,1) \\ (1,0) & \text { (b) } p=1, h=(-1,1,1) & (0,1) \\ (1,0) & (0,1) & (1,0)\end{array}$

(c) $p=-1, h=(1,-1,1)$.

Fig. 4. Rod structures for 3-centred single black holes with a central horizon

More generally, we see that a single $S^{3}$ black hole, so $p= \pm 1$, requires an odd number of centres. Such solutions correspond to a spherical black hole in a bubbling spacetime with $n-2$ bubbles (and 1 disc), or $n-3$ bubbles (and 2 discs), depending on which centre corresponds to the horizon, and have not been previously constructed.

Now consider single black hole solutions with $S^{1} \times S^{2}$ horizon topology, so $p=0$. These must have an even number of centres $n$ and from the above we see that there are $\frac{n}{2}\left(\begin{array}{c}n-1 \\ n / 2\end{array}\right)$ inequivalent $n$-centred solutions with a single black ring. For even $n>2$ we find there are an increasing number of inequivalent black ring in bubbling spacetime solutions which have not previously been discussed. For example, in Fig. 5, we list the six possible rod structures for 4-centred single black ring solutions.

5.4. Multi black hole solutions. We will not consider the case of multi black holes in detail. Previously constructed examples in this class are the multi black rings [38], a 


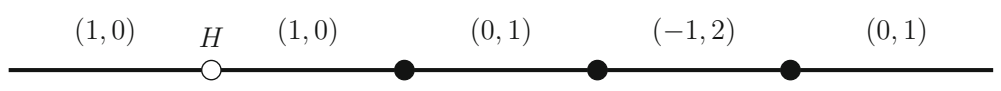

(a) $p=0, h=(0,1,1,-1)$

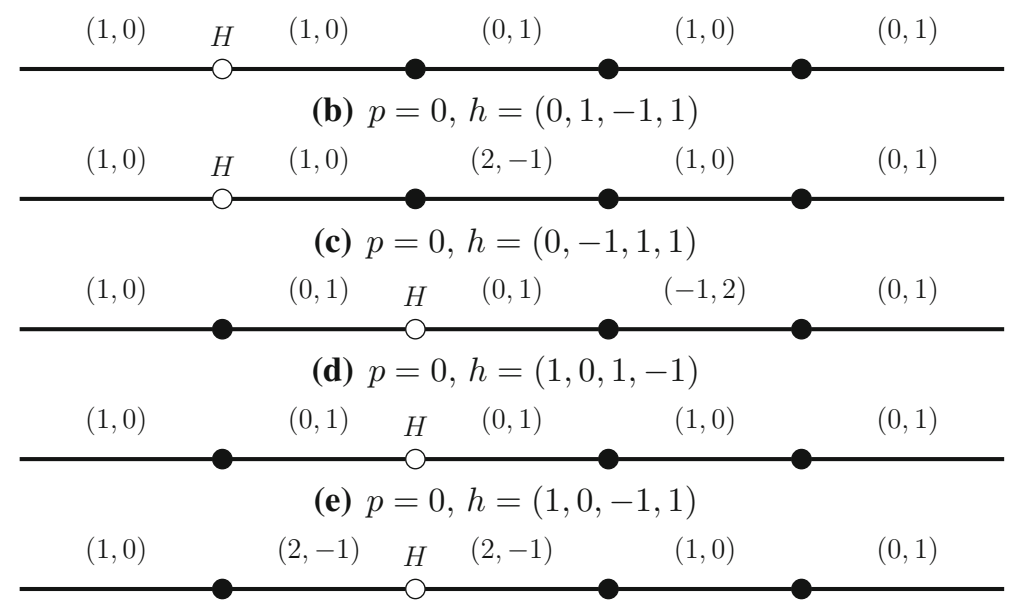

(f) $p=0, h=(-1,0,1,1)$

Fig. 5. Rod structures for 4-centred single black ring solutions

double $S^{3}$ black hole [48] and more generally multi black lenses [49]. We emphasise that the multi extreme Reissner-Nordstrom and multi BMPV black hole solutions [50] do not fit into our classification as they are not biaxisymmetric (they preserve at most $S O(3)$ rotational symmetry).

5.5. Physical properties. The mass and angular momenta for the general solution described in Theorem 5 are given by (using (158))

$$
\begin{aligned}
M & =3 \pi\left(\sum_{i=1}^{n} \ell_{i}+\frac{4}{9} m^{2}\right), \\
J_{\psi} & =2 \pi \sum_{i=1}^{n}\left(\frac{4}{9} m^{2} k_{i}-m \ell_{i}+m_{i}\right), \\
J_{\phi} & =2 \pi \sum_{i=1}^{n} z_{i}\left(m h_{i}+\frac{3}{2} k_{i}\right),
\end{aligned}
$$

and the electric charge

$$
Q=\frac{1}{4 \pi} \int_{S^{3}} \star F=2 \sqrt{3} \pi\left(\sum_{i=1}^{n} \ell_{i}+\frac{4}{9} m^{2}\right)
$$

satisfies the BPS bound $Q=\frac{2}{\sqrt{3}} M$. 
As noted above, the general solution possesses nontrivial topology in the form of 2-cycles (bubbles, discs, tubes) corresponding to the finite axis rods $I_{i}$, where $i=$ $1, \ldots, n-1$. The fluxes through these noncontractible 2-cycles $C_{i}$ are given by

$$
\Pi\left[C_{i}\right]=\frac{1}{4 \pi} \int_{C_{i}} F=\left.\frac{\sqrt{3}}{2} \frac{h_{j} m_{j}+\frac{1}{2} k_{j} \ell_{j}}{h_{j} \ell_{j}+k_{j}^{2}}\right|_{j=i} ^{j=i+1} .
$$

Note that for a corner $z_{j}$, the expression on the right hand side simplifies to $\left(h_{j} m_{j}+\right.$ $\left.\frac{1}{2} k_{j} \ell_{j}\right) /\left(h_{k} \ell_{j}+k_{j}^{2}\right)=-k_{j} / h_{j}$. The nontrivial topology also allows us to define constant magnetic potentials $\Phi_{i}$ associated with each axis rod $I_{i}$ by [5]

$$
\iota_{v_{i}} F=\mathrm{d} \Phi_{i}
$$

where we fix $\Phi_{i} \rightarrow 0$ asymptotically. We find that, for $i=1, \ldots, n-1$, the $\Phi_{i}$ evaluated on the corresponding axis rods are

$$
\left.q_{i} \equiv \Phi_{i}\right|_{I_{i}}=\frac{\sqrt{3}}{2}\left(\left(\left.\chi\right|_{I_{i}}-1\right) \sum_{j=1}^{i} k_{j}+\left(\left.\chi\right|_{I_{i}}+1\right) \sum_{j=i+1}^{n} k_{j}\right),
$$

which are indeed constants.

Thus a solution with $n$-centres carries the global charges $Q, J_{\psi}, J_{\phi}$ (with $M$ fixed by $Q$ ) and also $n-1$ local magnetic potentials $q_{i}$ (or magnetic fluxes $\Pi\left[C_{i}\right]$ ), leading to a total of $n+2$ physical charges. On the other hand, the dimension of the moduli space (171) for a solution with a single black hole is $n+1$ and for a soliton is $n-1$. Therefore, for a single black hole there the must be a single constraint on the $n+2$ physical parameters, whereas for a soliton there must be three such constraints.

The constraints on the physical parameters can be seen more explicitly as follows. Using the constraints on the parameters (160) one can show that

$$
J_{\phi}=-2 \pi \sum_{i=1}^{n} \sum_{j<i}\left(h_{i} m_{j}-m_{i} h_{j}-\frac{3}{2}\left(\ell_{i} k_{j}-k_{i} \ell_{j}\right)\right) .
$$

The gauge freedom (166) implies that we can always set $k_{j}=0$ for at least one $j \in$ $\{1, \ldots, n\}$ (since at least one $h_{i}$ must be nonvanishing due to (158)). Therefore, we may invert (186) to express the remaining $n-1$ parameters $k_{i \neq j}$ as linear combinations of the $n-1$ magnetic potentials $q_{i}$ (one can check the matrix relating the two sets of quantities is indeed invertible). This gives a direct physical interpretation to the parameters $k_{i}$. At every corner $z_{i}$ the parameters $\ell_{i}, m_{i}$ are determined in terms of $k_{i}$ and hence can also be expressed solely in terms of the magnetic potentials. In the case of a single black hole at position $z_{h}$, we can then invert (183) and (181) to express the parameters $\ell_{h}$ and $m_{h}$ purely in terms of the physical parameters,

$$
\ell_{h}=\ell_{h}\left(Q, q_{i}\right), \quad m_{h}=m_{h}\left(Q, J_{\psi}, q_{i}\right),
$$

and using these it is then clear that (187) implies the single constraint amongst the physical parameters is of the form

$$
J_{\phi}=J_{\phi}\left(Q, J_{\psi}, q_{i}\right)
$$


In the case of a soliton solution, all the parameters $\ell_{i}$ and $m_{i}$ are completely determined by the $k_{i}$ (and hence the $q_{i}$ ), which then implies the charge (183) and angular momenta $(181,187)$ can be expressed solely in terms of the magnetic potentials,

$$
Q=Q\left(q_{i}\right), \quad J_{\psi}=J_{\psi}\left(q_{i}\right), \quad J_{\phi}=J_{\phi}\left(q_{i}\right),
$$

thus giving three constraints on the physical parameters as anticipated above.

\section{Discussion}

In this work we have presented a complete classification of asymptotically flat, supersymmetric and biaxisymmetric solutions to five-dimensional minimal supergravity, which are regular on and outside an event horizon. Our analysis also covers the case of spacetimes containing no black hole, in which case we obtain a complete classification of soliton spacetimes in this class. The essential local result is that such solutions must be in the class of multi-centred Gibbons-Hawking solutions. Although these have been extensively studied over the last decade or so, a global analysis of these solutions has not been previously presented and therefore our work also fills this important gap. ${ }^{10} \mathrm{We}$ reveal a rich moduli space of $n$-centred solutions both with and without a black hole.

One of the main global results is that we find a refinement of the allowed horizon topologies in this class. That is, horizon cross-sections must be $S^{3}, S^{1} \times S^{2}$ or a lens space $L(p, 1)$, in particular ruling out $L(p, q)$ and $q \neq 1(\bmod p)$. Although examples of black hole solutions have been previously constructed for each possible type, we find that there are an infinite number of distinct black hole solutions for each of the horizon topologies. More precisely, the number of distinct $n$-centred solutions containing a single black hole grows rapidly with $n$ (see eqs. (178) and (179)).

An important technical problem which we were unable to solve is whether the constraints on the parameters of the solution required by smoothness of the horizon and the axes (given in Theorem 5) are in fact sufficient for smoothness and stably causality everywhere in the DOC. Based on numerical checks performed for the known examples we believe this is indeed the case, although this issue requires further investigation. Recently, progress in this direction for the bubbling soliton solutions was made [52]; it would be interesting to see if a similar method could be applied to the black hole case.

It is interesting to compare our results to vacuum gravity. Here the classification of asymptotically flat, stationary and biaxisymmetric spacetimes is an open problem. It is known that black holes in this class must have horizons of $S^{3}, S^{1} \times S^{2}$ or lens space $L(p, q)$ topology, however, it is not known whether a smooth solution exists for every possible rod structure. Indeed, the only known explicit solutions are the $S^{3}$ Myers-Perry black hole and the $S^{1} \times S^{2}$ black ring, both of which have the simplest possible rod structure. Given that we have found a refinement of the allowed horizon topologies for supersymmetric black holes, it is interesting to consider if this also happens for vacuum black holes. In fact we have shown that supersymmetry restricts the possible rod structures in such a way to constrain the horizon topology. In contrast, in the vacuum case, rod structures for black holes with $L(p, q)$ and $q \neq 1(\bmod p)$ are possible, although it is not known whether there exist corresponding smooth spacetimes [31]. It is therefore still possible that such topologies are also not realised for vacuum black holes, although this remains an open problem.

\footnotetext{
10 A global analysis of a subclass of supersymmetric solutions with a Gibbons-Hawking base which reduce to four-dimensional Euclidean Einstein-Maxwell solutions was performed in [51].
} 
On the other hand, if regular vacuum black holes with $L(p, q)$ and $q \neq 1(\bmod p)$ do exist, one then expects to be able to construct charged non-extremal versions of these in minimal supergravity and our results then show that such solutions would not have a supersymmetric limit. This is not what occurs for the known families of spherical black holes and black rings, where the supersymmetric case always arises as a limit case of a larger non-extremal family.

There are a number of possible directions in which our work could be extended. Clearly, a similar classification in the more general minimal supergravity coupled to an arbitrary number of vector multiplets could be carried out, where one anticipates analogous results. It would also be interesting to adapt our analysis to spacetimes with other relevant asymptotics such as Kaluza-Klein or Taub-NUT. Indeed, the local version of our horizon analysis, Theorem 2, could be applied directly in these cases.

It would be interesting to investigate the implications of our results for black hole non-uniqueness and the related problem of counting of black hole microstates in string theory. Recently it was shown that a black hole in a spacetime with a single bubble in the DOC may have the same conserved charges as the standard spherical BMPV black hole (thereby demonstrating continuous violation of uniqueness even for spherical black holes) [6]. Furthermore, it was also shown that this solution has higher entropy than the BMPV black hole as one approaches the BMPV upper spin limit [7]. Our classification presents the opportunity to analyse the full space of solutions with the same charges (and symmetry) as the standard solutions. We leave this interesting question to future work.

Acknowledgements. VB is supported by an EPSRC studentship. JL is supported by STFC [ST/L000458/1].

Open Access This article is distributed under the terms of the Creative Commons Attribution 4.0 International License (http://creativecommons.org/licenses/by/4.0/), which permits unrestricted use, distribution, and reproduction in any medium, provided you give appropriate credit to the original author(s) and the source, provide a link to the Creative Commons license, and indicate if changes were made.

\section{References}

1. Chrusciel, P.T., Lopes Costa, J., Heusler, M.: Stationary black holes: uniqueness and beyond. Living Rev. Rel. 15, 7 arXiv:1205.6112 (2012)

2. Emparan, R., Reall, H.S.: Black holes in higher dimensions. Living Rev. Rel. 11, 6 arXiv:0801.3471 (2008)

3. Bena, I., Warner, N.P.: Black holes, black rings and their microstates. Lect. Notes Phys. 755, 1 arXiv:hep-th/0701216 (2008)

4. Gibbons, G.W., Warner, N.P.: Global structure of five-dimensional fuzzballs. Class. Quant. Grav. 31, 025016 arXiv:1305.0957 (2014)

5. Kunduri, H.K., Lucietti, J.: The first law of soliton and black hole mechanics in five dimensions. Class. Quant. Grav. 31, 032001 arXiv:1310.4810 (2014)

6. Kunduri, H.K., Lucietti, J.: Black hole non-uniqueness via spacetime topology in five dimensions. JHEP 10, 082 arXiv:1407.8002 (2014)

7. Horowitz, G.T., Kunduri, H.K., Lucietti, J.: Comments on black holes in bubbling spacetimes. JHEP 06, 048 arXiv: 1704.04071 (2017)

8. Kunduri, H.K., Lucietti, J.: Supersymmetric black holes with lens-space topology. Phys. Rev. Lett. 113, 211101 arXiv:1408.6083 (2014)

9. Tomizawa, S.M. Nozawa: Supersymmetric black lenses in five dimensions. Phys. Review D. 94, 4 arXiv:1606.06643 (2016)

10. Hollands, S., Ishibashi, A.: Black hole uniqueness theorems in higher dimensional spacetimes. Class. Quant. Grav. 29, 163001 arXiv:1206.1164 (2012)

11. Friedman, J.L., Schleich, K., Witt, D.M.: Topological censorship. Phys. Rev. Lett. 71, 1486 arXiv:gr-qc/9305017, [Erratum: Phys. Rev. Lett.75,1872(1995)] (1993)

12. Galloway, G.J., Schoen, R.: A Generalization of Hawking's black hole topology theorem to higher dimensions. Commun. Math. Phys. 266, 571 arXiv:gr-qc/0509107 (2006)

13. Hollands, S., Ishibashi, A., Wald, R.M.: A Higher dimensional stationary rotating black hole must be axisymmetric. Commun. Math. Phys. 271, 699 arXiv:gr-qc/0605106 (2007) 
14. Gibbons, G.W., Ida, D., Shiromizu, T.: Uniqueness and nonuniqueness of static vacuum black holes in higher dimensions. Prog. Theor. Phys. Suppl. 148, 284 arXiv:gr-qc/0203004 (2003)

15. Gibbons, G.W., Ida, D., Shiromizu, T.: Uniqueness of (dilatonic) charged black holes and black p-branes in higher dimensions. Phys. Rev. D66, 044010 arXiv:hep-th/0206136 (2002)

16. Gibbons, G.W., Ida, D., Shiromizu, T.: Uniqueness and nonuniqueness of static black holes in higher dimensions. Phys. Rev. Lett. 89, 041101 arXiv:hep-th/0206049 (2002)

17. Kunduri, H.K., Lucietti, J.: No static bubbling spacetimes in higher dimensional Einstein-Maxwell theory. arXiv:1712.02668 (2017)

18. Emparan, R., Reall, H.S.: Generalized Weyl solutions. Phys. Rev. D65, 084025 arXiv:hep-th/0110258 (2002)

19. Harmark, T.: Stationary and axisymmetric solutions of higher-dimensional general relativity. Phys. Rev. D70, 124002 arXiv:hep-th/0408141 (2004)

20. Hollands, S., Yazadjiev, S.: Uniqueness theorem for 5-dimensional black holes with two axial Killing fields. Commun. Math. Phys. 283, 749 arXiv:0707.2775 (2008)

21. Hollands, S., Yazadjiev, S.: A Uniqueness theorem for stationary Kaluza-Klein black holes. Commun. Math. Phys. 302, 631 arXiv:0812.3036 (2011)

22. Hollands, S., Yazadjiev, S.: A Uniqueness theorem for 5-dimensional Einstein-Maxwell black holes. Class. Quant. Grav. 25, 095010 arXiv:0711.1722 (2008)

23. Tomizawa, S., Yasui, Y., Ishibashi, A.: Uniqueness theorem for charged dipole rings in five-dimensional minimal supergravity. Phys. Rev. D81, 084037 arXiv:0911.4309 (2010)

24. Armas, J., Harmark, T.: Uniqueness theorem for black hole space-times with multiple disconnected horizons. JHEP 05, 093 arXiv:0911.4654 (2010)

25. Yazadjiev, S.: Uniqueness and nonuniqueness of the stationary black holes in 5D Einstein-Maxwell and Einstein-Maxwell-dilaton gravity. JHEP 06, 083 arXiv:1104.0378 (2011)

26. Armas, J.: Uniqueness of black holes with bubbles in minimal supergravity. Class. Quant. Grav. 32, 045001 arXiv:1408.4567 (2015)

27. Yazadjiev, S.S., Nedkova, P.G.: Magnetized configurations with black holes and Kaluza-Klein bubbles: Smarr-like relations and first law. Phys. Rev. D80, 024005 arXiv:0904.3605 (2009)

28. Yazadjiev, S.S.: A Uniqueness theorem for black holes with Kaluza-Klein asymptotic in 5D EinsteinMaxwell gravity. Phys. Rev. D82, 024015 arXiv:1002.3954 (2010)

29. Haas, P.A.: Mass formula of a five-dimensional almost-BPS supergravity soliton with a magnetic "Bolt". arXiv:1511.02005 (2015)

30. Haas, P.: Topological sources of soliton mass and supersymmetry breaking. Class. Quantum Grav. 35 arXiv:1705.03992 (2017)

31. Khuri, M., Weinstein, G., Yamada, S.: Stationary vacuum black holes in 5 dimensions. arXiv:1711.05229 (2017)

32. Reall, H.S.: Higher dimensional black holes and supersymmetry. Phys. Rev. D68, 024024 arXiv:hep-th/0408141 [Erratum: Phys. Rev.D70,089902(2004)] (2003)

33. Gutowski, J.B.: Uniqueness of five-dimensional supersymmetric black holes. JHEP 08, 049 arXiv:hep-th/0404079 (2004)

34. Breckenridge, J.C., Myers, R.C., Peet, A.W., Vafa, C.: D-branes and spinning black holes. Phys. Lett. B391, 93 arXiv:hep-th/9602065 (1997)

35. Chrusciel, P.T., Reall, H.S., Tod, P.: On Israel-Wilson-Perjes black holes. Class. Quant. Grav. 23, 2519 arXiv:gr-qc/0512116 (2006)

36. Gauntlett, J.P., Gutowski, J.B., Hull, C.M., Pakis, S., Reall, H.S.: All supersymmetric solutions of minimal supergravity in five-dimensions. Class. Quant. Grav. 20, 4587 arXiv:hep-th/0209114 (2003)

37. Elvang, H., Emparan, R., Mateos, D., Reall, H.S.: A supersymmetric black ring. Phys. Rev. Lett. 93, 211302 arXiv:hep-th/0407065 (2004)

38. Gauntlett, J.P., Gutowski, J.B.: Concentric black rings. Phys. Rev. D71, 025013 arXiv:hep-th/0408010 (2005)

39. Bena, I., Warner, N.P.: Bubbling supertubes and foaming black holes. Phys. Rev. D74, 066001 arXiv:hep-th/0505166 (2006)

40. Berglund, P., Gimon, E.G., Levi, T.S.: Supergravity microstates for BPS black holes and black rings. JHEP 06, 007 arXiv:hep-th/0505167 (2006)

41. Chrusciel, P.T.: On higher dimensional black holes with abelian isometry group. J. Math. Phys. 50, 052501 arXiv:0812.3424 (2009)

42. Niehoff, B.E., Reall, H.S.: Evanescent ergosurfaces and ambipolar hyperkähler metrics. JHEP 04, 130 arXiv:1601.01898 (2016)

43. Gibbons, G.W., Ruback, P.J.: The hidden symmetries of multicenter metrics. Commun. Math. Phys. 115, 267 (1988)

44. Tomizawa, S., Yasui, Y., Ishibashi, A.: Uniqueness theorem for charged rotating black holes in fivedimensional minimal supergravity. Phys. Rev. D79, 124023 arXiv:0901.4724 (2009)

45. Figueras, P., Lucietti, J.: On the uniqueness of extremal vacuum black holes. Class. Quant. Grav. 27, 095001 arXiv:0906.5565 (2010) 
46. Kunduri, H.K., Lucietti, J.: Classification of near-horizon geometries of extremal black holes. Living Rev. Rel. 16, 8 arXiv:1306.2517 (2013)

47. Chrusciel, P.T., Nguyen, L.: A uniqueness theorem for degenerate Kerr-Newman black holes. Annales Henri Poincare 11, 585 arXiv:1002.1737 (2010)

48. Crichigno, P.M., Porri, F., Vandoren, S.: Bound states of spinning black holes in five dimensions. JHEP 05, 101 arXiv:1603.09729 (2017)

49. Tomizawa, S., Okuda, T.: Asymptotically flat multiblack lenses. Phys. Rev. D 95, 064021 arXiv:1701.06402 (2017)

50. Gauntlett, J.P., Myers, R.C., Townsend, P.K.: Black holes of D = 5 supergravity. Class. Quant. Grav. 16, 1 arXiv:hep-th/9810204 (1999)

51. Dunajski, M., Hartnoll, S.A.: Einstein-Maxwell gravitational instantons and five dimensional solitonic strings. Class. Quant. Grav. 24, 1841 arXiv:hep-th/0610261 (2007)

52. Avila, J., Ramirez, P.F., Ruiperez, A.: One thousand and one bubbles. arXiv:1709.03985 (2017)

Communicated by P. Chrusciel 